ARTICLE

\title{
A microRNA program regulates the balance between cardiomyocyte hyperplasia and hypertrophy and stimulates cardiac regeneration
}

\author{
Andrea Raso 1,12, Ellen Dirkx 1,12, Vasco Sampaio-Pinto 1,2, Hamid el Azzouzi,3, Ryan J. Cubero (i) 4,5, \\ Daniel W. Sorensen ${ }^{6}$, Lara Ottaviani ${ }^{1}$, Servé Olieslagers ${ }^{1}$, Manon M. Huibers ${ }^{7}{ }^{7}$, Roel de Weger ${ }^{7}$, Sailay Siddiqi ${ }^{8}$, \\ Silvia Moimas (10 9 , Consuelo Torrini ${ }^{9}$, Lorena Zentillin9', Luca Braga9 , Diana S. Nascimento (1) ${ }^{2}$, \\ Paula A. da Costa Martins (10) 1,10, Jop H. van Berlo (D) ${ }^{6}$, Serena Zacchigna (iD ${ }^{8}$, Mauro Giacca (i) ${ }^{9,11}$ \& \\ Leon J. De Windt (1) ${ }^{1 \times}$
}

Myocardial regeneration is restricted to early postnatal life, when mammalian cardiomyocytes still retain the ability to proliferate. The molecular cues that induce cell cycle arrest of neonatal cardiomyocytes towards terminally differentiated adult heart muscle cells remain obscure. Here we report that the miR-106b 25 cluster is higher expressed in the early postnatal myocardium and decreases in expression towards adulthood, especially under conditions of overload, and orchestrates the transition of cardiomyocyte hyperplasia towards cell cycle arrest and hypertrophy by virtue of its targetome. In line, gene delivery of miR106b 25 to the mouse heart provokes cardiomyocyte proliferation by targeting a network of negative cell cycle regulators including E2f5, Cdkn1c, Ccne1 and Wee1. Conversely, genetargeted miR-106b 25 null mice display spontaneous hypertrophic remodeling and exaggerated remodeling to overload by derepression of the prohypertrophic transcription factors Hand2 and Mef2d. Taking advantage of the regulatory function of miR-106b 25 on cardiomyocyte hyperplasia and hypertrophy, viral gene delivery of miR-106b 25 provokes nearly complete regeneration of the adult myocardium after ischemic injury. Our data demonstrate that exploitation of conserved molecular programs can enhance the regenerative capacity of the injured heart.

\footnotetext{
${ }^{1}$ Department of Molecular Genetics, Faculty of Science and Engineering, Faculty of Health, Medicine and Life Sciences, Maastricht University, Maastricht, The Netherlands. ${ }^{2}$ i3S - Instituto de Investigação e Inovação em Saúde, INEB - Instituto Nacional de Engenharia Biomédica, ICBAS - Instituto de Ciências Biomédicas de Abel Salazar, University of Porto, Porto, Portugal. ${ }^{3}$ Department of Molecular Genetics, Erasmus University MC, Rotterdam, The Netherlands. ${ }^{4}$ The Abdus Salam International Centre for Theoretical Physics, Trieste, Italy. ${ }^{5}$ IST Austria, Klosterneuburg, Austria. ${ }^{6}$ Stem Cell Institute and Lillehei Heart Institute, Department of Medicine, University of Minnesota, Minneapolis, MN, USA. ${ }^{7}$ Department of Pathology, University Medical Center Utrecht, Utrecht, The Netherlands. ${ }^{8}$ Department of Cardiothoracic Surgery, Radboud University Medical Center, Nijmegen, The Netherlands. ${ }^{9}$ International Centre for Genetic Engineering and Biotechnology (ICGEB), Trieste, Italy. ${ }^{10}$ Department of Physiology and Cardiothoracic Surgery, Faculty of Medicine, University of Porto, Porto, Portugal. ${ }^{11}$ School of Cardiovascular Medicine and Sciences, King's College London, London, UK. ${ }^{12}$ These authors contributed equally: Andrea

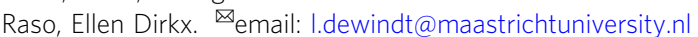


$\mathrm{P}$ roliferation of cardiogenic precursor cells and division of immature cardiomyocytes is key to mammalian cardiac morphogenesis during embryonic and fetal development ${ }^{1,2}$. The neonatal mammalian heart still retains considerable proliferative capacity reminiscent of lower vertebrates ${ }^{3-7}$, and is sustained by hypoxic conditions ${ }^{8}$, activation of Hippo signaling 9 , various transcriptional regulators ${ }^{10-12}$, and endogenous microRNA mechanisms ${ }^{13,14}$. Complete regeneration of the injured myocardium, including the human neonatal heart ${ }^{15}$, exclusively takes place in a short time window during fetal and early postnatal life, when cardiomyocytes still possess the ability to proliferate $^{7}$. Shortly after birth, however, the majority of cardiomyocytes lose their proliferative capacity, and shift toward a terminally differentiated phenotype, with restricted ability to reactivate mitosis ${ }^{16}$. Limited myocyte turnover does occur in the adult mammalian heart, but insufficient to restore contractile function following injury ${ }^{17}$. The default growth response of the adult heart to overload or injury consists primarily of cardiomyocyte hypertrophy, a form of cellular growth without cell division that often precipitates in heart failure ${ }^{18}$, a serious clinical disorder that represents a primary cause of morbidity and hospitalization. In humans, despite remarkable progresses made by device-based therapies and drug interventions ${ }^{19}$, the only way to replace lost cardiomyocytes is heart transplantation. Identification of the developmental molecular mechanisms that stimulate a proliferative phenotype in early postnatal life, control cell cycle arrest, and produce a hypertrophic response of the terminally differentiated heart muscle, may hold the key to unlock the regenerative potential of the adult mammalian heart.

\section{Results}

Suppression of the $m i R-106 b \sim 25$ cluster facilitates cardiac remodeling. We recently demonstrated that mature miR-25 transcripts are downregulated in two mouse models of heart failure, and results in the derepression of the bHLH transcription factor Hand 2 in the postnatal mammalian myocardium ${ }^{20}$. miR-25 is embedded in the $m i R-106 b \sim 25$ cluster, located on mouse chromosome 5 (chromosome 7 in humans) in an intronic region of the DNA-replication gene $\mathrm{Mcm} 7$ and consists of three miRNAs: $m i R-106 b$, $m i R-93$, and $m i R-25$ (Fig. 1a). Here we show that each $m i R-106 b \sim 25$ cluster member displayed decreased expression in human cardiac biopsies of end-stage heart failure obtained upon heart transplantation compared to healthy controls (Fig. 1b), decreased expression in the calcineurin transgenic mouse model of heart failure (Supplementary Fig. 1a) and in pressure overloaded mouse hearts in both the early and late remodeling phase (Supplementary Fig. 1b, c).

To more directly address the ramifications of reduced expression of $m i R-106 b \sim 25$ for the postnatal heart, we subjected cohorts of miR-106b 25-/- mice ${ }^{21}$, where expression of the cluster members was undetectable in the heart (Supplementary Fig. 1d), to sham surgery or pressure overload by transverse aortic constriction (TAC) surgery and serially assessed cardiac geometry and function at four weeks (Fig. 1c). Remarkably, miR-106b 25-/- mice already suffered from a mild form of eccentric hypertrophy at baseline as evidenced in sham-operated mice by an increased cardiac geometry, reduced wall thickness, reduced ejection fraction (EF), and increased cardiomyocyte size at four weeks after sham surgery (Fig. 1d-k; Table 1). Four weeks of TAC surgery in wild-type mice or $m i R-106 b \sim 25-1-$ mice resulted in severe myocyte disarray, interstitial fibrosis, increased heart weight, left ventricular dilation, systolic and diastolic dysfunction as well as a transcript induction of Nppa, Nppb, Acta1, and Myh7 as hypertrophic "stress" markers with consistently more severe phenotypes in $m i R-106 b \sim 25-/-$ mice (Fig. 1d-k; Table 1). In line, silencing of the individual
miR-106b 25 cluster members with specific antagomirs (Supplementary Fig. 2a, b) resulted in spontaneous and mild eccentric remodeling as evidenced by an increased cardiac geometry (Supplementary Fig. 2c), abnormal echocardiographic parameters (Supplementary Fig. 2d-g), increased cardiomyocyte size (Supplementary Fig. 2h) and induction of hypertrophic markers (Supplementary Fig. 2j), albeit milder compared to the phenotype of $m i R$ 106b 25-I- mice, supporting the conclusion that the individual cluster members work in a concerted fashion.

Mechanistically, we have previously shown $m i R$-25-dependent regulation of the prohypertrophic embryonic transcription factor Hand2 in the adult heart and could repeat this finding (Supplementary Fig. $2 \mathrm{k}, 1)^{20}$. To further understand the mechanistic role of $m i R-106 b \sim 25$ in cardiac remodeling, we analyzed bioinformatics databases to search for $m i R-106 b$, $m i R-93$, and $m i R-25$ binding sites in cardiac expressed protein-coding transcripts. We identified a perfect match for a $m i R-25$ heptametrical seed sequence in the transcription factor Myocyte enhancer factor $2 \mathrm{~d}(\mathrm{Mef} 2 \mathrm{~d})$ that showed complete evolutionary conservation among vertebrates (Fig. 11), validated the target gene using luciferase reporters harboring either the wild-type or sitedirected mutagenesis of key nucleotides in the miR-25 binding site (Fig. $1 \mathrm{~m}$ ), and finally demonstrated derepression of Mef2d protein expression upon $m i R-25$ silencing in cardiomyocytes by western blotting (Fig. 1n). Taken together, these data demonstrate that the miR-106b 25 cluster is repressed in the failing heart and causes eccentric hypertrophic remodeling by simultaneous derepression of the prohypertrophic transcription factors Hand2 and Mef2d.

Activation of the $m i R-106 b \sim 25$ cluster provokes cardiac enlargement. To further evaluate the function of the miR$106 b \sim 25$ cluster in cardiac disease, we measured the expression of $m i R-106 b, m i R-93$, and $m i R-25$ in the postnatal mouse heart at postnatal day $0(\mathrm{p} 0), \mathrm{p} 5, \mathrm{p} 10, \mathrm{p} 15$, and p20, representing developmental stages toward adulthood (p56, week 8). The data demonstrate that the miR-106b 25 cluster was on average 6-8 fold higher expressed early in the postnatal phase and slowly decreased in expression to adult expression levels after which expression remained stable (Fig. 2a). In line, $m i R-106 b \sim 25$ expression in the adult heart muscle cells was very low compared to adult non-myocyte cells that still retain the ability to proliferate (Supplementary Fig. 3).

Next, we made use of the high cardiac tropism and prolonged expression of serotype 9 adeno-associated viral (AAV9) vectors following systemic delivery ${ }^{22}$. AAV9 vectors expressing $m m u$ $m i R-106 b, m m u-m i R-93$, and $m m u-m i R-25$ precursor miRNAs (AAV9-miR-106b 25), or a control vector with an empty multiple cloning site (AAV9-MCS), were injected intraperitoneally in neonatal mice at postnatal day 1 (p1; Fig. 2b) as a gain-offunction approach (Fig. 2c). Anticipating a cardiac phenotype resistant to hypertrophic remodeling, much to our surprise, at 4 weeks, the hearts of mice injected with AAV9-miR-106b 25 were histologically normal but significantly enlarged (Fig. 2d, e). Echocardiographic analysis demonstrated that heart size and interventricular septum thickness in systole was significantly increased (Fig. 2e-i, Table 2), but cardiac morphometric dimensions and contractile function were normal as evidenced by the absence of ventricular dilation (Fig. 2h) and ejection fraction (Fig. 2i). There was no sign of inflammatory cell infiltration or cardiac fibrosis (Fig. 2d, j). Even more peculiar, individual myocyte size was unaltered and there was no evidence for reactivation of a "fetal" gene expression pattern characteristic for hypertrophic cardiomyocytes (Fig. 2k, 1). Conclusively, maintaining high $m i R-106 b \sim 25$ expression levels as observed in 

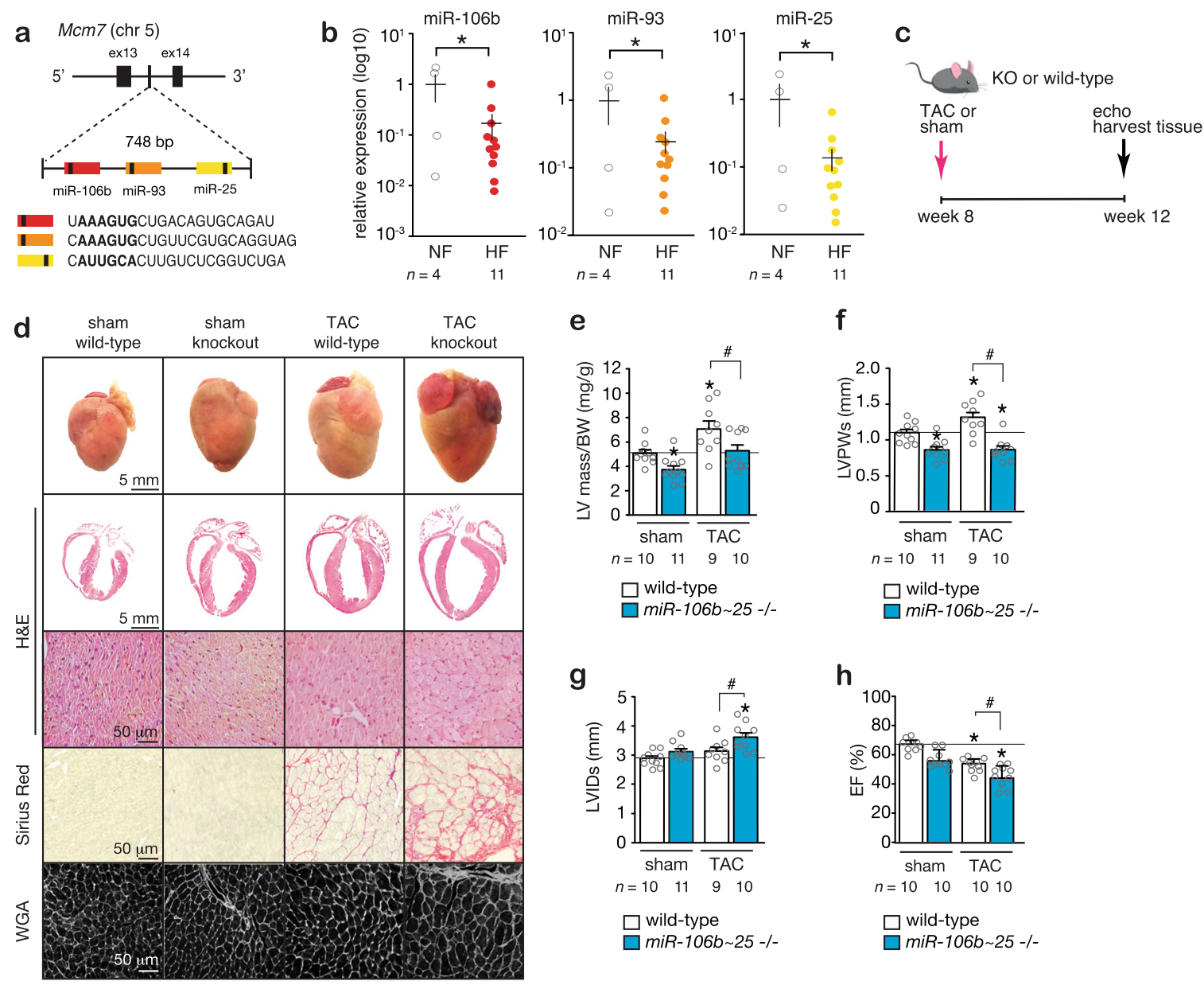

e

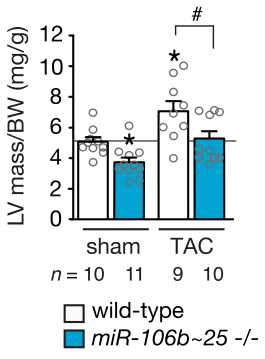

f

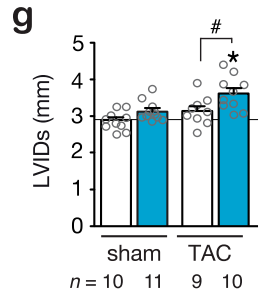

h
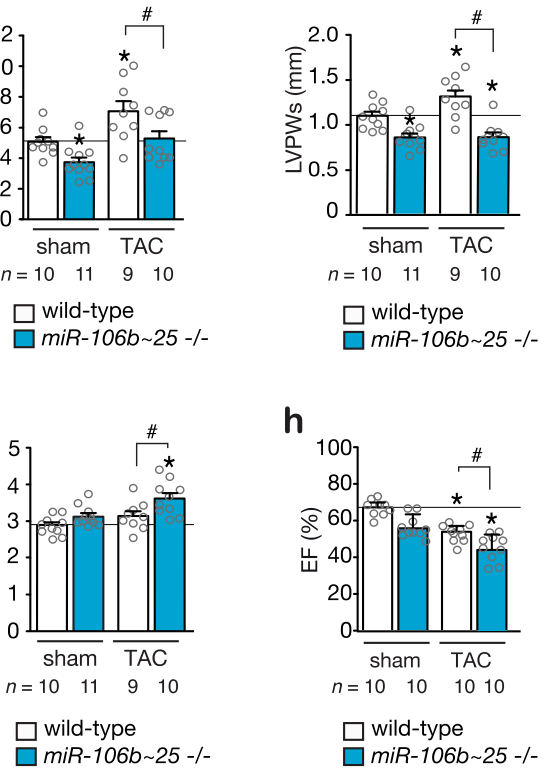

$\square$ wild-type

$\square$ miR-106b 25 -/-
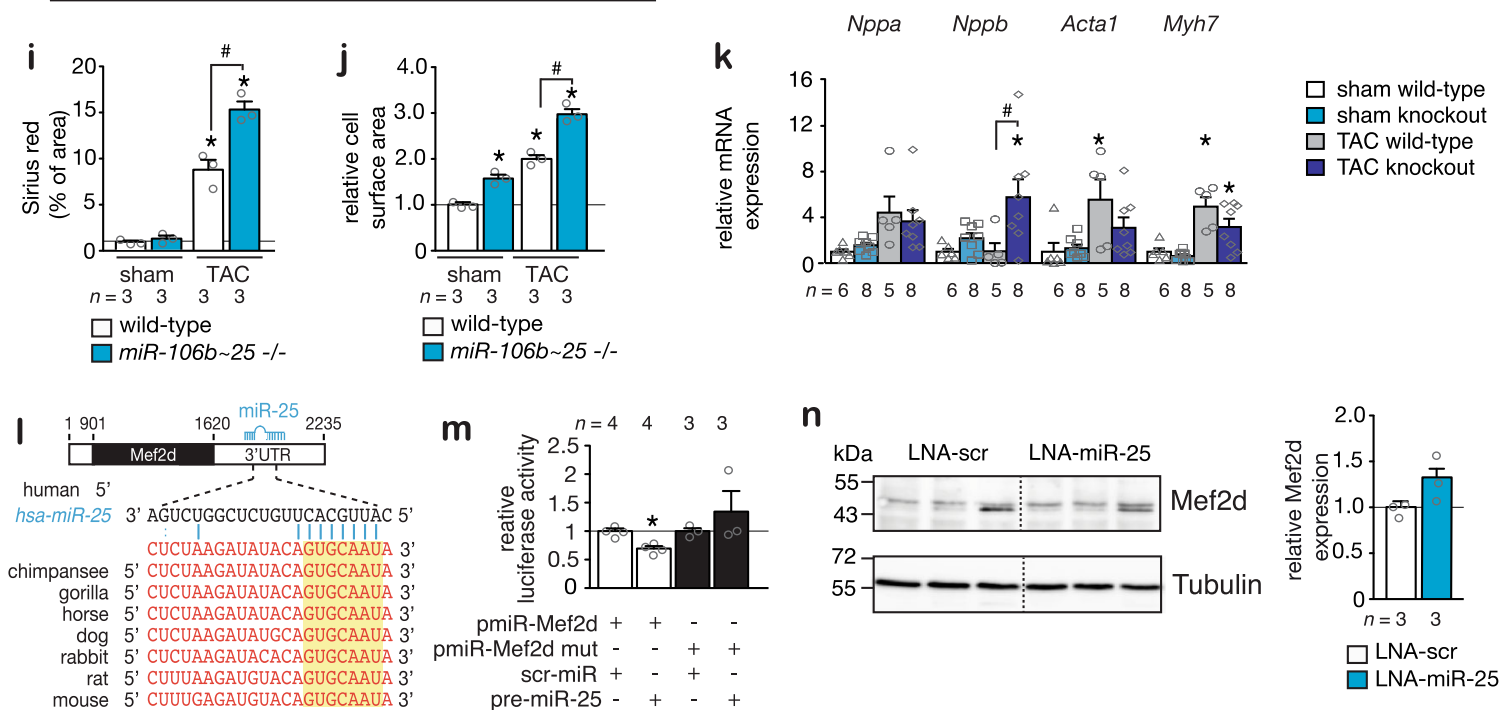

Fig. 1 miR-106b 25 gene deletion induces hypertrophic cardiac remodeling. a A schematic representation of the mouse $M \mathrm{~cm} 7$ gene harboring the miR106b 25 cluster in intron 13. b Real-time PCR analysis of miR-106b, miR-93, and miR-25 abundance in human non-failing or failing myocardium. c Design of the study. d Representative images of whole hearts (top panels), haematoxylin \& eosin (H\&E)-stained sections of four-chamber view (second panel), high magnification H\&E sections (third panel), Sirius Red stained sections (fourth panel), and wheat germ agglutinin (WGA)-stained (fifth panel) histological sections. Quantification of e left ventricular mass/body weight (BW) ratio, $\mathbf{f}$ left ventricular posterior wall thickness in systole (LVPWs), $\mathbf{g}$ Left ventricular internal diameter in systole (LVIDs), $\mathbf{h}$ ejection fraction (EF). $\mathbf{i}$ Quantification of the fibrotic area by Sirius Red staining and $\mathbf{j}$ cell surface areas by wheat germ agglutinin (WGA) staining. $\mathbf{k}$ Real-time PCR analysis of Nppa, Nppb, Acta1, and Myh7, $n$ refers to number of animals. I Location and evolutionary conservation of hsa-miR-25 seed region on Mef2d. $\mathbf{m}$ Activity assay of luciferase reporter constructs shows the binding of hsa-miR-25 to the $3^{\prime} \mathrm{UTR}$ of Mef2d, $n$ refers to number of transfection experiments. $\mathbf{n}$ Western blot analysis of endogenous Mef2d and GAPDH as a loading control in hearts from wild type (WT) versus miR-106b 25 knock-out (KO) mice, $n$ refers to number of hearts. ${ }^{\star} P<0.05$ vs corresponding control group; ${ }^{\#} P<0.05$ vs corresponding treatment (error bars are s.e.m.). Statistical analysis consisted of a two-tailed Student's t-test (b, $\mathbf{m}, \mathbf{n}$ ) or a One-way ANOVA followed by Dunnett multiple comparison test (e-k). Source data are provided as a Source data file. 
Table 1 Morphometric and echocardiographic characteristics of WT versus miR-106b-25 KO mice subjected to sham or TAC surgery for 4 weeks.

\begin{tabular}{lllll} 
& Sham & & TAC \\
& WT & KO & WT & KO \\
\hline$n$ & 10 & 11 & 9 & 10 \\
BW (g) & $21.2 \pm 0.2$ & $21.3 \pm 0.4$ & $21.0 \pm 0.5$ & $21.0 \pm 0.4$ \\
LV mass (mg) & $107 \pm 5$ & $80 \pm 5^{\star}$ & $145 \pm 12^{\star}$ & $110 \pm 8^{\#}$ \\
LV mass/BW (mg/g) & $5.1 \pm 0.3$ & $3.8 \pm 0.4^{\star}$ & $7.1 \pm 0.7^{\star}$ & $5.3 \pm 0.5^{\#}$ \\
IVSd (mm) & $0.83 \pm 0.05$ & $0.68 \pm 0.03^{\star}$ & $1.04 \pm 0.06^{\star}$ & $0.89 \pm 0.04^{\#}$ \\
IVSs (mm) & $1.16 \pm 0.06$ & $1.04 \pm 0.03$ & $1.40 \pm 0.07^{\star}$ & $1.16 \pm 0.05^{\#}$ \\
LVIDd (mm) & $4.09 \pm 0.08$ & $4.15 \pm 0.09$ & $4.10 \pm 0.14$ & $4.41 \pm 0.11$ \\
LVIDs (mm) & $2.87 \pm 0.08$ & $3.14 \pm 0.08$ & $3.14 \pm 0.07$ & $3.61 \pm 0.15^{\star \#}$ \\
LVPWd (mm) & $0.89 \pm 0.04$ & $0.65 \pm 0.04^{\star}$ & $1.04 \pm 0.06^{\star}$ & $0.70 \pm 0.05^{\star \#}$ \\
LVPWs (mm) & $1.11 \pm 0.05$ & $0.83 \pm 0.03^{\star}$ & $1.30 \pm 0.08^{\star}$ & $0.88 \pm 0.05^{\star \#}$ \\
EF (\%) & $64 \pm 1$ & $57 \pm 1^{\star}$ & $54 \pm 2^{\star}$ & $45 \pm 3^{\star} \#$ \\
FS (\%) & $29 \pm 1$ & $25 \pm 1^{\star}$ & $23 \pm 1^{\star}$ & $18 \pm 1^{\star} \#$ \\
E/A (mm/s) & $1.73 \pm 0.11$ & $1.53 \pm 0.10$ & $1.62 \pm 0.10$ & $1.57 \pm 0.24^{\star}$ \\
\hline
\end{tabular}

Data are expressed as means \pm SEM.

BW body weight, LV left ventricular, IVSd interventricular septal thickness at end-diastole, IVSs interventricular septal thickness at end-systole, LVIDd left ventricular internal dimension at end-diastole, LVIDs left ventricular internal dimension at end-systole, LVPwd left ventricular posterior wall thickness at end-diastole, LVPws left ventricular posterior wall thickness at end-systole, EF ejection fraction, FS fractional shortening, E/A Doppler E/A ratio.

*Indicates $P<0.05$ vs sham group subjected to treatment with a control antagomir.

\#Indicates $P<0.05$ vs experimental group.

the early postnatal developmental period produced cardiac enlargement in the adult heart without classical signs of pathological hypertrophic remodeling.

The $m i R-106 b \sim 25$ cluster stimulates cardiomyocyte proliferation. To understand how the miR-106b 25 cluster can evoke cardiac growth, we resorted to cardiomyocyte cultures isolated from neonatal hearts, which retain both hypertrophic and proliferative properties ${ }^{23}$. We performed a fluorescence-microscopybased analysis in neonatal rat cardiomyocytes transfected with either a control precursor miRNA, or precursors for $m i R-106 b$, $m i R-93$ or $m i R-25$. At $72 \mathrm{~h}$, cells were stained for sarcomeric $\alpha-$ actinin to distinguish cardiomyocytes from non-myocytes, and we included the proliferation marker 5-ethynyl-2'-deoxyuridine (EdU), a thymidine analog that is incorporated into newly synthesized DNA (Fig. 3a, b). Automated image segmentation and analysis was performed to selectively quantify number of proliferating cardiomyocytes ( $\alpha$-actinin,+ EdU+; Fig. $3 c$ ) and total number of cardiomyocytes (a-actinin+; Fig. 3d; Supplementary Fig. 4a). The data demonstrate that $m i R-106 b$, $m i R-93$, or $m i R-25$ stimulated cardiomyocyte proliferation and cardiomyocyte numbers by a factor of 3 with no substantial differences between the miRNA cluster members.

Next, to evaluate whether the $m i R-106 b \sim 25$ cluster would also enhance cardiomyocyte proliferation in vivo, we injected AAV9miR-106b 25 intraperitoneally in neonatal mice at p1 to elevate cardiac miR-106b 25 cluster expression, administered Edu intraperitoneally at p10 and analyzed the hearts at p12 (Fig. 3e). The hearts of p12 mice injected with AAV9-106b 25 were significantly enlarged compared to those from mice injected with the control AAV9 (Fig. 3f), with no sign of inflammatory cell infiltration or increased cardiac fibrosis content, and no increase in cardiomyocyte size (Fig. 3f, g). Confocal microscopy indicated that the number of both cardiomyocytes in $S$ phase of the cell cycle (a-actinin +, EdU+; Fig. 3h, i; Supplementary Fig. 1e) and mitotic cardiomyocytes (a-actinin +, PH3+; Fig. 3h, j; a-actinin + , Aurora B+; Supplementary Fig. 4b) was significantly increased in hearts of animals injected with AAV9-miR-106b 25 compared to hearts of animals injected with the control AAV9-MCS.

Next, to ascertain if the individual cluster members showed differences in activating cardiomyocyte proliferation, we also generated AAV9 vectors expressing either $m i R-106 b$, $m i R-93$, or miR-25 and administered the viral vectors intraperitoneally in neonatal mice at $\mathrm{p} 1$, administered EdU at p10 and analyzed the hearts at p12 (Supplementary Fig. 5a). In each case, mice with increased expression of either $m i R-106 b, m i R-93$, or $m i R-25$ were significantly enlarged (Supplementary Fig. 5b), showed no increase in cardiomyocyte cell size (Supplementary Fig. 5b, c), demonstrated an increased number of cardiomyocytes in $S$ phase of the cell cycle ( $\alpha$-actinin+, EdU+; Supplementary Fig. 5d, e) and mitotic cardiomyocytes ( $\alpha$-actinin,$+ \mathrm{PH} 3+$; Supplementary Fig. 5f, g; or a-actinin+, Aurora B+; Supplementary Fig. 5h).

Next, we employed stereology to simultaneously assess left ventricular volumes, $\mathrm{CM}$ volumes, $\mathrm{CM}$ nuclei densities, $\mathrm{CM}$ nucleation, and CM proliferation as described previously ${ }^{24-26}$ in wild-type neonatal mice, wild-type mice injected with AAV9miR-106b 25, and miR-106b 25 knockout mice from birth (p0) to $\mathrm{p} 12$, since CM proliferation is still abundant in early postnatal life in the mouse and essentially absent after p15 ${ }^{24}$ (Fig. 4a). CM nuclei in tissue sections were stained for the cardiomyocyte nuclear marker pericentriolar material 1 (PCM-1), CM multinucleation was determined by co-staining with wheat germ agglutinin (WGA) to delineate cell boundaries and PCM-1 to mark CM nuclei and CM proliferation assessed by co-labeling sections with PCM-1 and EdU (Fig. 4b). LV volumes increased from $5.5 \pm 0.4 \mathrm{~mm}^{3}$ to $13.2 \pm 1.0 \mathrm{~mm}^{3}$ at $\mathrm{p} 6$ and p12, respectively, with a tendency for mice that received AAV9-miR-106b 25 to have a slightly higher LV volume (Fig. 4c). The ratio of mono- to binucleated cardiomyocytes changed substantially during the first 12 postnatal days. On p6, a minority of cardiomyocytes $(14.1 \pm$ $1.1 \%)$ were binucleated, whereas at p12 the majority of cardiomyocytes became binucleated $(66.1 \pm 1.1 \%)$, with mice that received AAV9-miR-106b 25 having a slight reduction in the percentage of binucleated cardiomyocytes at p12 (61.2 $\pm 2.2 \%$; Fig. 4d). The total number of cardiomyocytes at p12 was considerably larger in mice that received AAV9-miR-106b 25 as were the number of EdU+ cardiomyocytes at p6 and p12 (Fig. 4e, f). In addition, the number of EdU+ cardiomyocytes was lower at p12 in miR-106b 25 knockout mice, indicating a requirement of the endogenous $m i R-106 b \sim 25$ cluster for cardiomyocyte proliferation in juvenile hearts (Fig. 4f). 
a

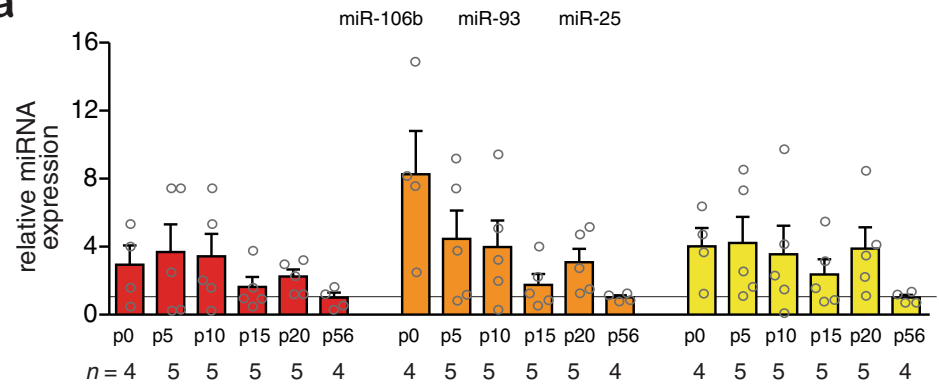

C
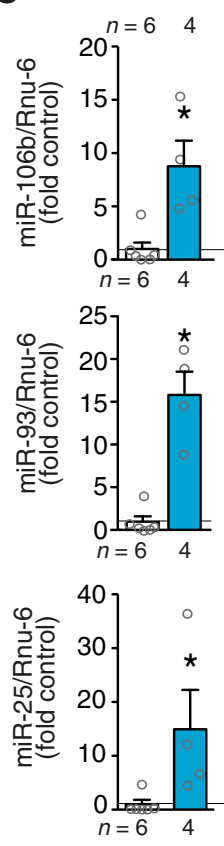

AAV9-MCS

AAV9-miR-106b 25
AAV9-

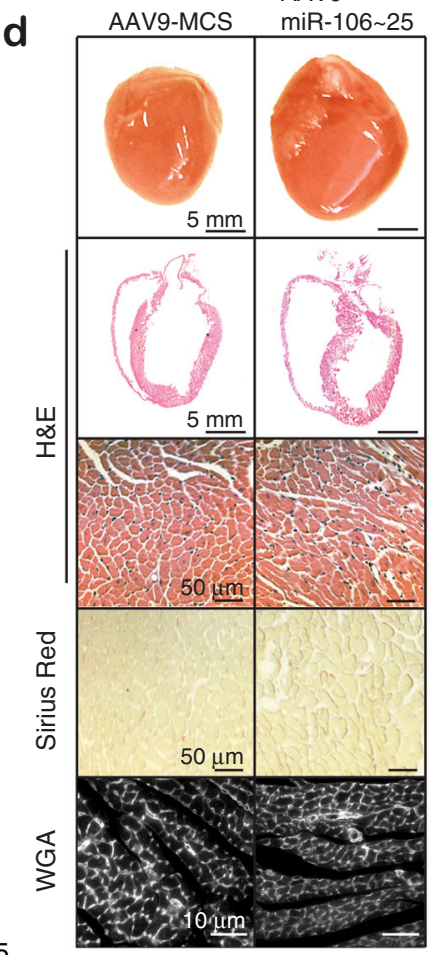

b

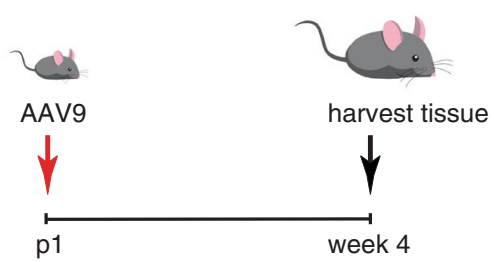

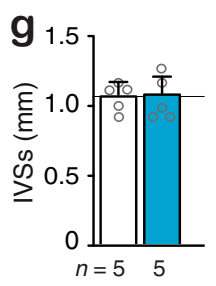
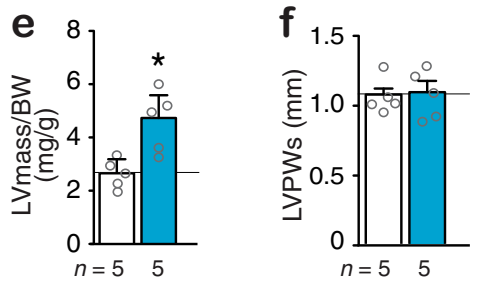

AAV9-MCS

$\square$ AAV9-miR-106b 25
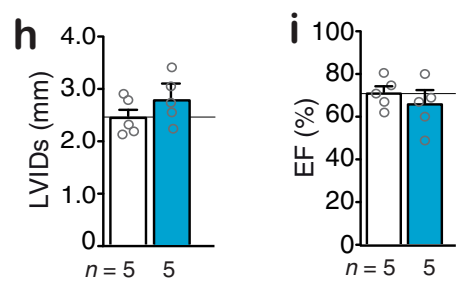

AAV9-MCS

AAV9-miR-106b 25

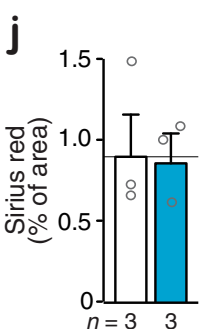

$\square$ AAV9-MCS

AAV9-miR-106b 25
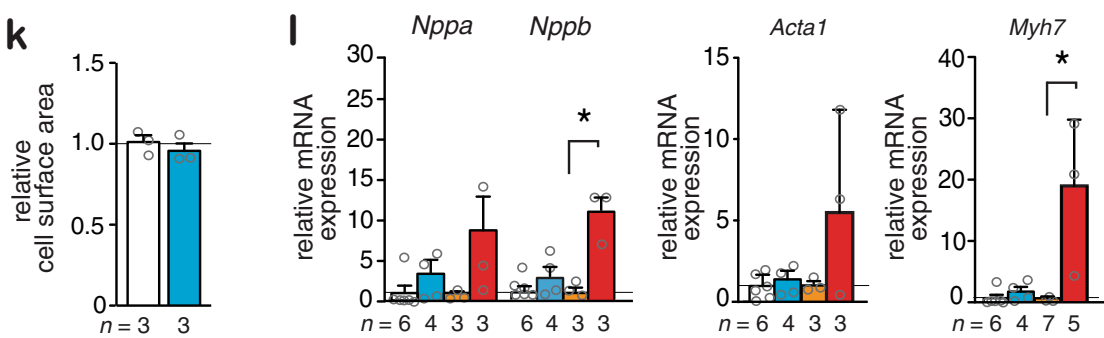

$\square$ AAV9-MCS

$\square$ AAV9-miR-106b 25

Fig. 2 Overexpression of the miR-106b 25 cluster induces cardiac growth with sustained function. a Real-time PCR analysis of miR-106b, miR-93, and miR-25 abundance in mouse hearts at postnatal day 0 (p0), p5, p10, p15, p20, and p56 (2 months) of age. b Design of the study. c Real-time PCR analysis of miR-106b, miR-93, and miR-25 abundance in AAV9-MCS versus AAV9-miR-106b 25 hearts, 4 weeks after injection. $\mathbf{d}$ Representative images of whole hearts (top panels), H\&E-stained sections of four-chamber view (second panel), high magnification H\&E sections (third panel), Sirius Red stained sections (fourth panel), and WGA-stained (fifth panel) sections. Quantification of $\mathbf{e}$ LV/BW ratio, $\mathbf{f} L V P W s, \mathbf{g} I V S s, \mathbf{h} L V I D s$, and $\mathbf{i}$ EF of mice that received AAV9MCS or AAV9-miR-106b 25. Quantification of $\mathbf{j}$ the fibrotic area by Sirius Red staining and $\mathbf{k}$ the cell surface areas by WGA-staining. I Real-time PCR analysis of Nppa, Nppb, Acta1, and Myh7 in hearts from mice that received AAV9-MCS or AAV9-miR-106b 25; or WT or calcineurin transgenic (Myh6$\mathrm{CnA}$ ) mice, a mouse model for heart failure, $n$ refers to number of animals. ${ }^{\star} P<0.05$ vs corresponding control group (error bars are s.e.m.). Statistical analysis consisted of a two-tailed Student's $t$-test (c, e-k) or a One-way ANOVA followed by Dunnett multiple comparison test (I). Source data are provided as a Source data file. 
Table 2 Morphometric and echocardiographic characteristics of mice treated for 4 weeks with AAV9-MCS or AAV9-miR-106b 25.

\begin{tabular}{lll} 
& AAV9-MCS & AAV9-miR-106b -25 \\
\hline$n$ & 5 & 5 \\
BW (g) & $29.4 \pm 0.5$ & $24.3 \pm 1.7$ \\
LV mass (mg) & $79 \pm 7$ & $121 \pm 26^{\star}$ \\
LV mass/BW (mg/g) & $2.7 \pm 0.2$ & $4.8 \pm 0.6^{\star}$ \\
IVSd (mm) & $0.68 \pm 0.03$ & $0.91 \pm 0.08^{\star}$ \\
IVSs (mm) & $1.05 \pm 0.04$ & $1.28 \pm 0.09$ \\
LVIDd (mm) & $3.75 \pm 0.12$ & $4.08 \pm 0.08^{\star}$ \\
LVIDs (mm) & $2.50 \pm 0.14$ & $2.78 \pm 0.19$ \\
LVPWd (mm) & $0.84 \pm 0.09$ & $0.85 \pm 0.08$ \\
LVPWs (mm) & $1.07 \pm 0.06$ & $1.09 \pm 0.07$ \\
EF (\%) & $70 \pm 3$ & $66 \pm 5$ \\
FS (\%) & $33 \pm 2$ & $31 \pm 3$ \\
\hline
\end{tabular}

Data are expressed as means \pm SEM.

$B W$ body weight, $L V$ left ventricular, IVSd interventricular septal thickness at end-diastole, IVSs interventricular septal thickness at end-systole, LVIDd left ventricular internal dimension at enddiastole, LVIDs left ventricular internal dimension at end-systole, LVPwd left ventricular posterior wall thickness at end-diastole LVPws left ventricular posterior wall thickness at end-systole, EF ejection fraction, FS fractional shortening, E/A Doppler E/A ratio.

*Indicates $P<0.05$ vs sham group subjected to treatment with a control antagomir. *Indicates $P<0.05$ vs sham group subjected to
\#Indicates $P<0.05$ vs experimental group.

Taken together, these results show that elevated cardiac expression of the complete $m i R-106 b \sim 25$ cluster or the individual cluster members significantly enhance proliferation of at least a subset of cardiomyocytes in vivo.

The miR-106b 25 cluster suppress cell cycle inhibitors. To elucidate the molecular mechanisms underlying the proliferative effects of this microRNA cluster, we performed RNA-seq to assess the transcriptome changes in neonatal rat cardiomyocyte RNA after transfection with $m i R-106 b$, miR-93, or $m i R-25$ mimics. This analysis identified 1082 genes for $m i R-106 b, 1347$ genes for $m i R$ 93, and 1673 genes for $m i R-25$ upregulated and 1253 genes for $m i R-106 b, 1594$ genes for $m i R-93$, and 2327 genes for $m i R-25$ downregulated (at 1.0 reads per kilobase of exon model per million mapped reads (RPKM) cutoff and 1.30 fold-change cutoff; Fig. 5a, Supplementary Table S1). We then imposed bioinformatic predictions of miRNA seed sequence interactions with rat transcripts that were downregulated by the miRNAs upon transfection to cardiomyocytes according to the transcriptomic data, yielding 112,217 , and $420 m i R-106 b, m i R-93$, and $m i R-25$ target genes respectively, with substantial overlap between the $m i R-106 b$ and miR-93 targetome (Fig. 5b). Next, a bioinformatic protein-protein interaction network was derived that integrates and scores protein interactions across different evidence channels (conserved neighborhood, co-occurrence, fusion, co-expression, experiments, databases, and text mining). For each miRNA in the miR106b 25 cluster, a subnetwork was extracted. Analysis of the networks showed a particularly dense and often overlapping enrichment for genes functioning in cell cycle regulation, and to a lesser extent networks of genes involved in actin cytoskeletal organization, oxidative stress, and components of the Hippo pathway (Fig. 5c).

$m i R-106 b$ and miR-93 had overlapping targets among the cyclins and cyclin-dependent kinases including Ccnb1 (cyclin B), Ccna2 (cyclin A), Ccnd1 and Ccnd2 (cyclin Ds) as well as Ccne2 (cyclin E), suggesting that these two miRNAs affected the G2phase of the cell cycle (Fig. 5d). miR-25 showed an upregulation of Ccnd1 and Ccnd2 (cyclin Ds) accompanied by a downregulation of Ccne1 and Ccne2 (cyclin E), Ccna2 (cyclin A) and Ccnb1 (cyclin B), suggesting that this miRNA affected the Sphase of the cell cycle (Fig. 5d). Apart from regulation of specific
Cyclin/Cdk complexes, common targets among all members of the miR-106b 25 cluster included various cell cycle inhibitors that act on various cell cycle phases, including Cdkn1a (cyclindependent kinase inhibitor $1 \mathrm{a}$ or $\mathrm{p} 21^{\mathrm{CIP}} 1$ ), Cdkn1c (cyclindependent kinase inhibitor $1 \mathrm{c}$ or $\mathrm{p} 57^{\mathrm{KIP} 2}$ ), the retinoblastoma transcriptional corepressor 1 (Rb1) inhibitor E2F5, and the G2 checkpoint kinase Weel (Fig. 5c, d).

We independently validated our results with an unbiased highcontent screen in neonatal rat cardiomyocytes transfected with siRNAs against $18 \mathrm{miR}-106 \mathrm{~b} 25$ targetome members to screen for their individual contribution to cardiomyocyte proliferation. The data demonstrate that treatment siRNAs against individual targetome members induced only a partial increase in CM proliferation compared to that observed with $m i R-106 b \sim 25$ overexpression, indicating that the effect of the miRNA cluster probably results from a cumulative effect on multiple, cellular mRNA targets (Fig. 5e, f). Finally, we identified perfect matches for the heptametrical seed sequence for the miRNAs in the $3^{\prime}$ UTRs of E2F5 and Cdkn1c that showed evolutionary conservation among vertebrates (Supplementary Fig. 6a, c), validated the target genes using luciferase reporters harboring either the wildtype or site-directed mutagenesis of key nucleotides in the miRNA binding sites (Supplementary Fig. 6b, d), and finally demonstrated derepression of E2F5, Cdkn1c, Wee1 and Ccne1 protein expression in cultured cardiomyocytes transfected with an antimiR for $m i R-106 b, m i R-25$ or in hearts from $m i R-106 b \sim 25$ null mice by western blotting (Supplementary Fig. 6e-g).

Collectively, these data demonstrate that the miR-106b 25 cluster regulates densely overlapping networks of genes involved in cell cycle regulation and a number of key cell cycle inhibitors, explaining the proliferative effects of this miRNA cluster.

The $m i R-106 b \sim 25$ cluster stimulates post-infarction cardiac regeneration. The adult heart is characterized by a very poor regenerative potential. Given that the $m i R-106 b \sim 25$ cluster is higher expressed in the early postnatal phase and regulates cell cycle regulators and postnatal cardiomyocyte proliferation, we hypothesized that viral delivery of the miRNA cluster could potentially enhance post-infarction regeneration in the adult heart. To test this, adult CD1 mice underwent permanent ligation of left anterior descending (LAD) coronary artery to induce myocardial infarction (MI) and hearts were injected in the periinfarcted area with AAV9-miR-106 25 or a control AAV9 vector (AAV-MCS) (Fig. 6a). This approach resulted in efficient overexpression of each miRNA over control expression levels after MI (Fig. 6b). At 3 weeks of MI, cross-sectioning hearts from ligation to base demonstrated that hearts injected with the control AAV9 vector displayed the typical large and thinned scarred infarct accompanied with severe biventricular dilation (Fig. 6c, d). In sharp contrast, hearts injected with AAV9-miR-106 25 demonstrated significantly reduced infarct size and preservation of viable LV tissue and cardiac geometry (Fig. 6c, d). The echocardiographic analysis demonstrated near complete normalization of LV mass (Table 3), LVPWd (Fig. 6e), LVIDs (Fig. 6f), LVEF (Fig. 6g) and other functional parameters (Table 3), and a reduction in "stress" marker genes (Supplementary Fig. 6h). Confocal microscopy revealed that mice that received AAV9miR-106b 25 at three weeks displayed a significant number of EdU-positive cardiomyocyte nuclei in the infarct zone (Fig. 6h) with well-integrated cardiomyocytes within the myocardial structure indicative of active proliferation and regeneration following infarction.

Finally, we cross-bred tamoxifen-inducible genetic lineagetracing Myh6-MerCreMer mice to a Rosa26 tdTomato reporter mice to permanently mark Myh6-expressing cells (i.e., 
a

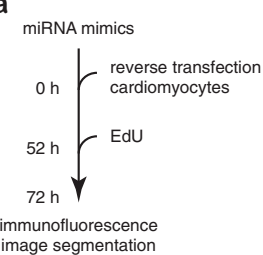

mage segmentation b

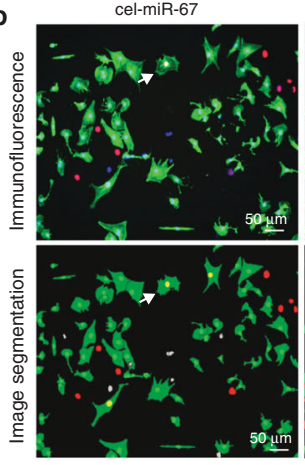

f

e

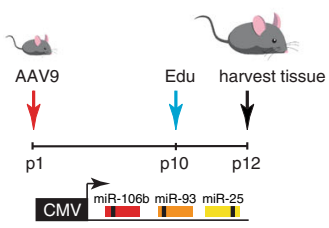

g

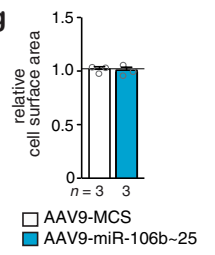

miR-106b

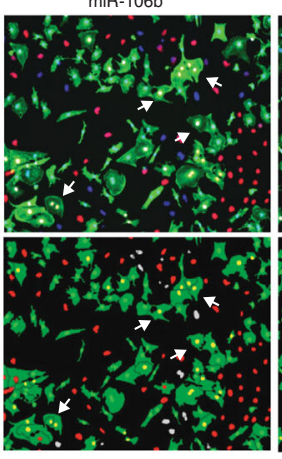

h
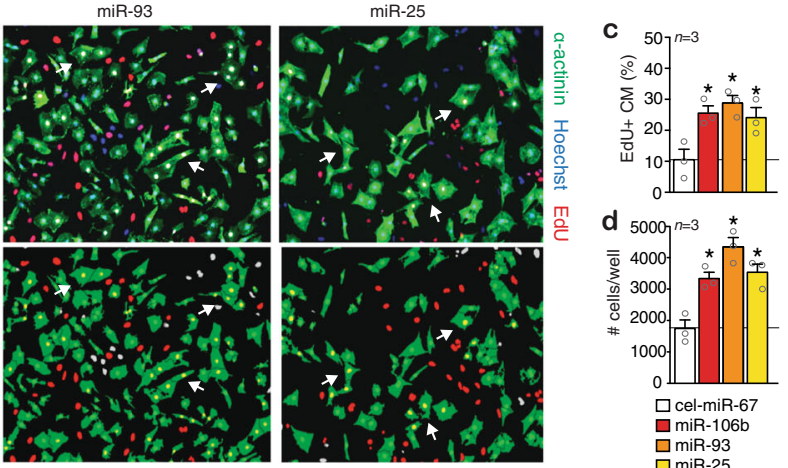

d 5000

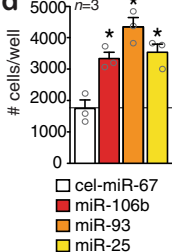

AAV9-MCS

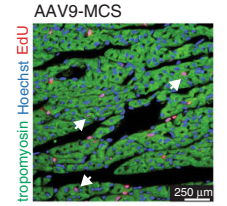

AAV9-miR-106

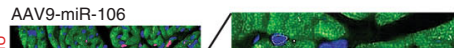

AAV9-miR-106b 25

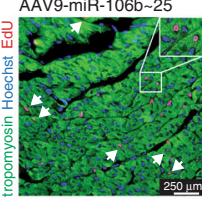

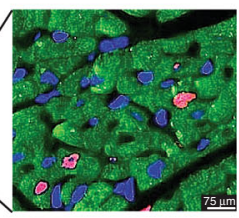

AAV9-miR-93
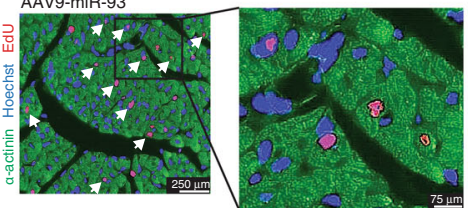
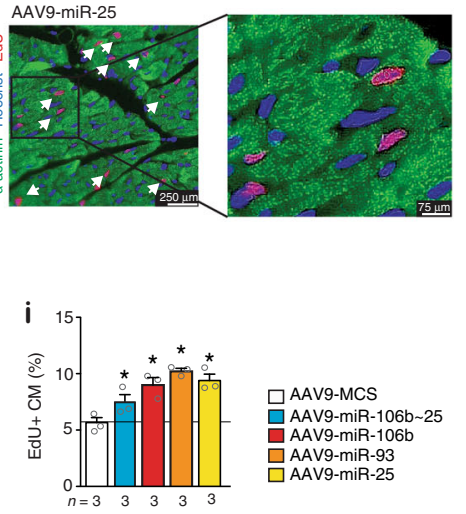

$\square$ AAV9-MCS

$\square$ AAV9-miR-106b 25

AAV9-miR-106b
AAV9-miR-93

$\square$ AAV9-miR-25

Fig. 3 Overexpression of the $\mathbf{m i R}-\mathbf{1 0 6} \boldsymbol{b} \sim \mathbf{2 5}$ cluster induces cardiomyocyte (CM) proliferation. $\mathbf{a}$ Workflow of the experiment. $\mathbf{b}$ Representative fluorescent microscopic images of rat CMs transfected with a control precursor miRNA, or precursors for miR-106b, miR-93, or miR-25 and stained for $\alpha$ actinin, 5-ethynyl-2'-deoxyuridine (EdU), and Hoechst. Quantification of $\mathbf{c}$ the number of proliferating $\mathrm{CMs}(\alpha$-actinin+, EdU+) and $\mathbf{d}$ total number of CMs $(\alpha$-actinin + ) after transfection with a control miRNA, or miR-106b, miR-93, or miR-25, $n$ refers to the number of transfection experiments. e Workflow of the

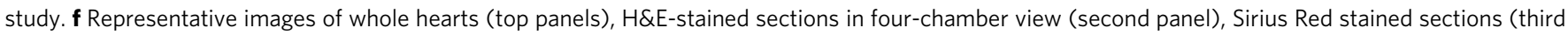

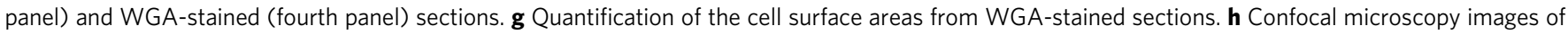
heart sections of mice treated with AAV9-MCS or AAV9-miR-106b 25 and stained for $\alpha$-actinin, EdU and Hoechst or $\alpha$-actinin, pH3 and Hoechst.

Quantification of $\mathbf{i}$ the number of proliferating $\mathrm{CMs}(\alpha$-actinin,$+ \mathrm{EdU}+$ ) and $\mathbf{j}$ the number of phospho-histone $3(\mathrm{pH} 3)$ positive $\mathrm{CMs}(\alpha-$ actinin + , $\mathrm{pH} 3+$ ), $n$ refers to number of hearts. ${ }^{\star} P<0.05$ vs corresponding control group (error bars are s.e.m.). Statistical analysis consisted of a two-tailed Student's $t$-test (g) or a One-way ANOVA followed by Dunnett multiple comparison test (c, d, i). Source data are provided as a Source data file.

cardiomyocytes) and follow the fate of their cellular descendants in vivo. Accordingly, adult Myh6-MCMR26tdTomato animals were treated with tamoxifen daily for 5 days to label cardiomyocytes with tdTomato. Next, Myh6-MCMR26tdTomato mice underwent permanent ligation of left anterior descending (LAD) coronary artery to induce MI and hearts were injected in the periinfarcted area with AAV9-miR-106 25 or a control AAV9 vector. A week before sacrifice, all animals received Edu to mark nuclei that are in $S$ phase of the cell cycle and we analyzed EdU $+/$ tdTomato + cells in tissue sections (Fig. 6i). As expected, tdTomato was exclusively expressed in cardiomyocytes. Importantly, the number of EdU+/tdTomato + cells in post-infarcted hearts that received AAV9-miR-106 25 was doubled compared to post-infarcted hearts that received a control AAV9 vector. This genetic lineage tracing approach confirms that the AAV9-miR106 25 vector stimulated the proliferation of pre-existing cardiomyocytes (Fig. 6i-k).

Conclusively, the $m i R-106 b \sim 25$ cluster, relatively high expressed in the early postnatal myocardium that still retains regenerative potential, directs networks of cell cycle regulators and stimulates proliferation of at least a subset of cardiomyocytes in vivo. In adulthood, the relative low cardiac expression of $m i R$ - 106b 25 sustains derepression of prohypertrophic cardiomyocyte gene programs that facilitate adverse remodeling in response to overload (Fig. 61). Exploiting this endogenous regulator between cardiomyocyte hyperplasia and hypertrophy by viral gene delivery enhances the endogenous regenerative capacity of the mammalian myocardium.

\section{Discussion}

Upon after birth, cardiomyocytes enter cell cycle arrest and become terminally differentiated accompanied by polyploidy and hypertrophy as the default growth response to overload or injury, 7,28 . This terminally differentiated phenotype and reduced cellular plasticity makes the heart more vulnerable in situations when increased workload is required as it either triggers irreversible cell death or hypertrophy ${ }^{29}$, which often precipitates in heart failure, a serious clinical disorder that represents the primary cause of morbidity and hospitalization in Western societies.

Here we report on the evolutionarily conserved microRNA cluster that is highly expressed in the early postnatal myocardium and repressed in the adult heart in man and mouse under disease conditions. Remarkably, $m i R-106 b \sim 25$ deficient mice as well as 
a
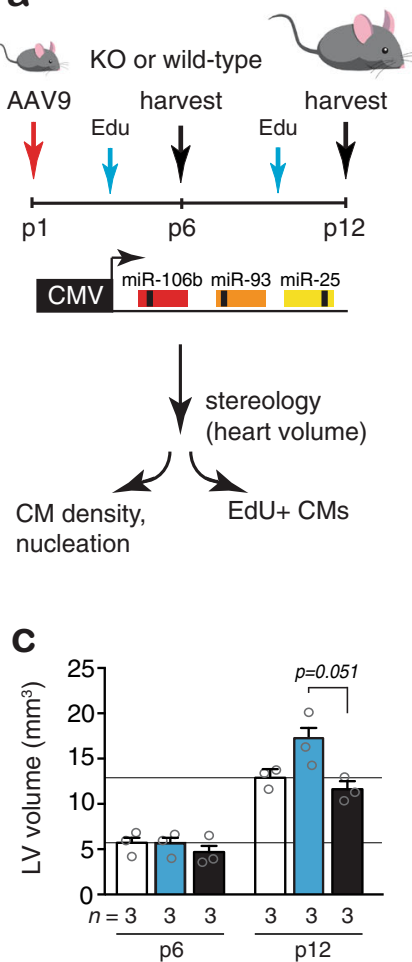

b
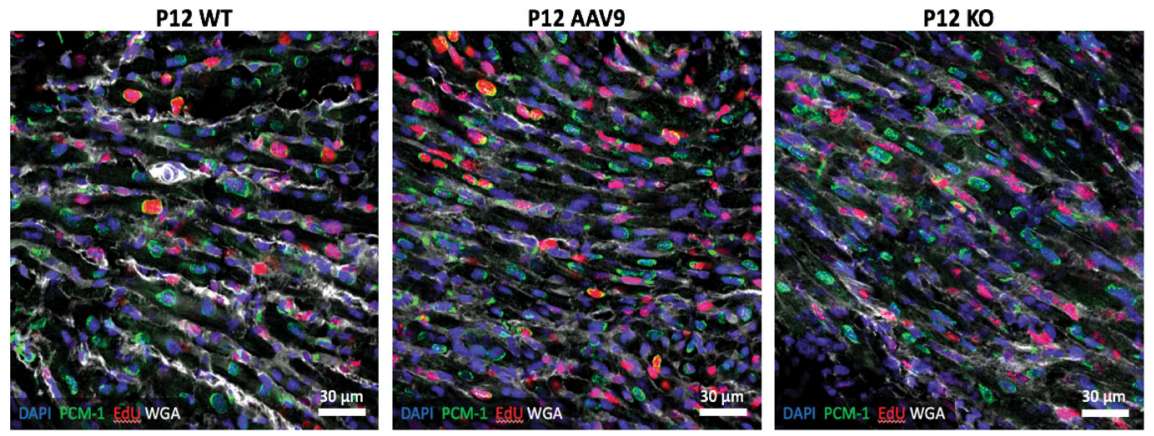
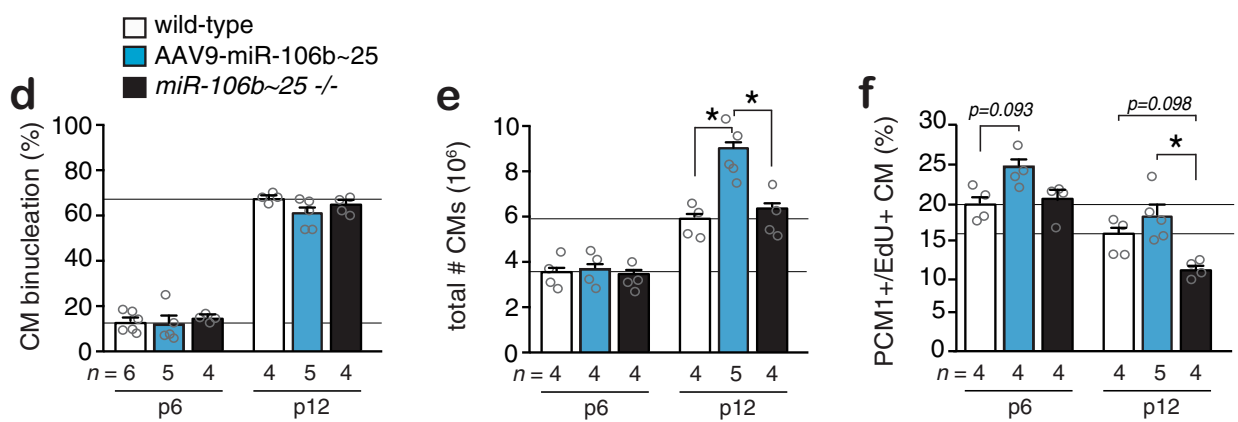

Fig. 4 The miR-106b $\mathbf{2 5}$ cluster controls the total number of CMs. a Workflow of the study. $\mathbf{b}$ Representative confocal images of sections co-stained for pericentriolar material 1 (PCM-1), 4',6-diamidino-2-phenylindole (DAPI), EdU, and WGA to assess PCM-1+EdU+ CMs in the experimental groups at p12. Quantification of $\mathbf{c}$ LV volumes, $\mathbf{d}$ percentage CM binucleation, e total number of CMs and $\mathbf{f}$ proliferating (PCM-1+, EdU+) CMs at p6 and p12 in the experimental groups, $n$ refers to number of hearts. ${ }^{\star} P<0.05$ vs corresponding control group (error bars are s.e.m.). Statistical analysis consisted of a Oneway ANOVA followed by Dunnett multiple comparison test (c-f). Source data are provided as a Source data file.

mice receiving antagomirs for either $m i R-106 b, m i R-93$, or $m i R$ 25 , display spontaneous cardiomyocyte hypertrophy and eccentric remodeling, mechanistically explained by the derepression of prohypertrophic downstream targets, most notably the bHLH transcription factor Hand $2^{20}$, as well as myocyte enhancer factor$2 \mathrm{~d}(\operatorname{Mef} 2 d)$, which serves as a terminal branch of stress signaling pathways that drive pathological cardiac remodeling ${ }^{30}$.

Overexpression of the $m i R-106 b \sim 25$ cluster, or the individual cluster members $m i R-106 b, m i R-93$, or $m i R-25$, by adenoassociated viral (AAV) vectors, stimulated cardiomyocyte proliferation, at least in a subset of cardiomyocytes, by targeting a network of genes with cell cycle regulatory functions including the key cell cycle inhibitors E2f5, Cdkn1c, Ccne1, and Wee1, positive cell cycle regulators that are abundantly expressed in the fetal and neonatal heart ${ }^{31,32}$. In the adult heart, cyclin-dependent kinase inhibitors, negative regulators of the cell cycle, are more prevalent $^{31,32}$. In line, forced overexpression of cyclin D2, a positive regulator of the G1/S transition, induced DNA synthesis, and proliferation in mammalian cardiomyocytes ${ }^{31,32}$. Additionally, overexpression of cyclin A2, which promotes the G1/S and $\mathrm{G} 2 / \mathrm{M}$ transitions, results in cardiomyocyte proliferation ${ }^{33}$, improved cardiac function after ischemic injury in mice ${ }^{34}$ and pigs $^{35}$.

That members of the miR-106b 25 cluster can evoke cardiomyocyte proliferation is confirmed by an unbiased, high-content screen to identify proliferative microRNAs ${ }^{36}$, while more recently, $m i R-25$ was demonstrated to provoke cardiomyocyte proliferation in zebrafish by repressing the cell cycle inhibitor Cdknc1 and tumor suppressor Lats $2^{37}$. Our results also revealed components of the Hippo/Yap pathway as miR-106b 25 targetome members. Hippo signaling has been widely studied in the context of cardiac regeneration ${ }^{38,39}$. In line, embryonic overexpression of Yap in mice induces hyperproliferation of cardiomyocytes and severely disproportional ventricles and death ${ }^{38,40,41}$, while forced expression of Yap in the adult heart provokes cardiomyocyte cell cycle re-entry and regeneration postinfarction injury ${ }^{40,42}$. However, unrestrained Yap activation may also display unwanted effects in pressure overloaded hearts due to cardiomyocyte dedifferentiation ${ }^{43}$.

Interestingly, contradicting effects of $m i R-25$ in the rodent heart have been reported. Some reports indicate that inhibition of miR-25 expression can lead to derepression of the target gene Serca $2 a$ and improve cardiac function ${ }^{44}$, and others report protection against oxidative stress or apoptosis induced by sepsis ${ }^{45,46}$. In contrast, others report that overexpression of $m i R-$ 25 is innocuous and induces proliferation by altering cell cycle genes in zebrafish ${ }^{37}$, while here we report cardiac enlargement secondary to enhanced cardiomyocyte proliferation, which at first sight could be misinterpreted as a pathological phenotype. From a therapeutic perspective, $m i R-25$ loss-of-function approaches have also shown disparate results from improving contractility on the one side $\mathrm{e}^{44}$, or inducing high blood pressure ${ }^{47}$, atrial fibrillation ${ }^{48}$, eccentric remodeling, and dysfunction ${ }^{20,47}$, on the other. It should be noted that distinct chemistries of antisense oligonucleotides can show quite different specificity or even cause sideeffects that may explain the opposing observations ${ }^{49,50}$. To avoid the uncertainty surrounding the use of oligonucleotide chemistries, here we resorted to an unequivocal gene deletion strategy where miR-106b 25 null mice display pathological cardiomyocyte hypertrophy, fibrosis, cardiac dilation and dysfunction, phenotypes that were recapitulated when silencing the individual cluster members with a $2^{\prime}$ Ome antisense chemistry. Using the same gene 
a

d
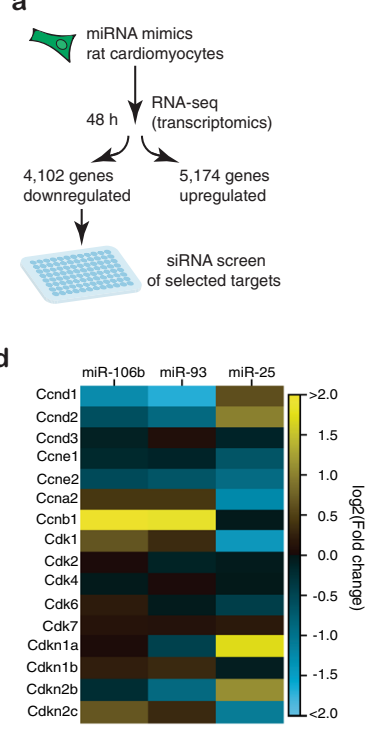

c

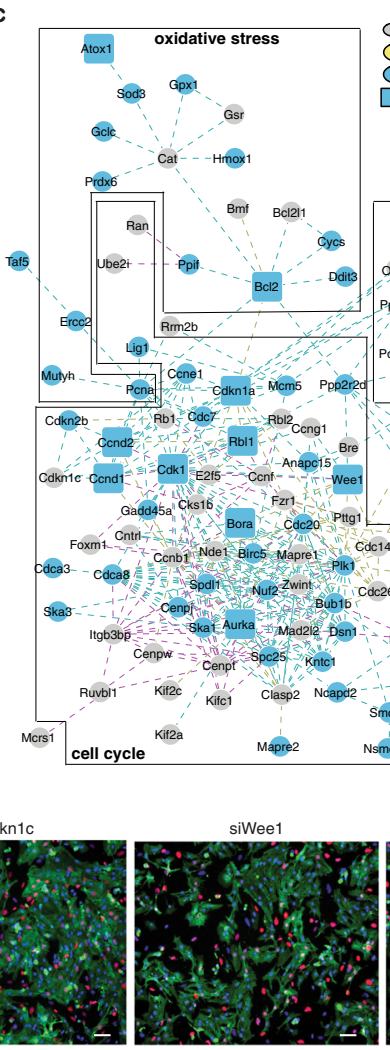

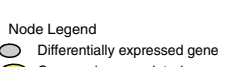
Differentially expressed gene commonly upregulated gene
commonly downregulated gene
commonly downregulated target Edge Legend ---- miR-106b network miR-93 network
miR-25 network
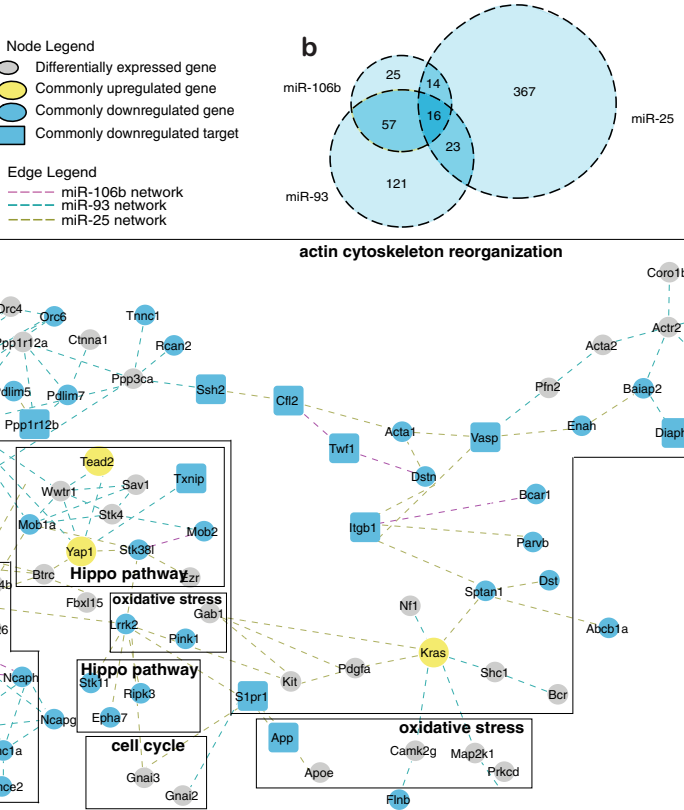

siTaok1
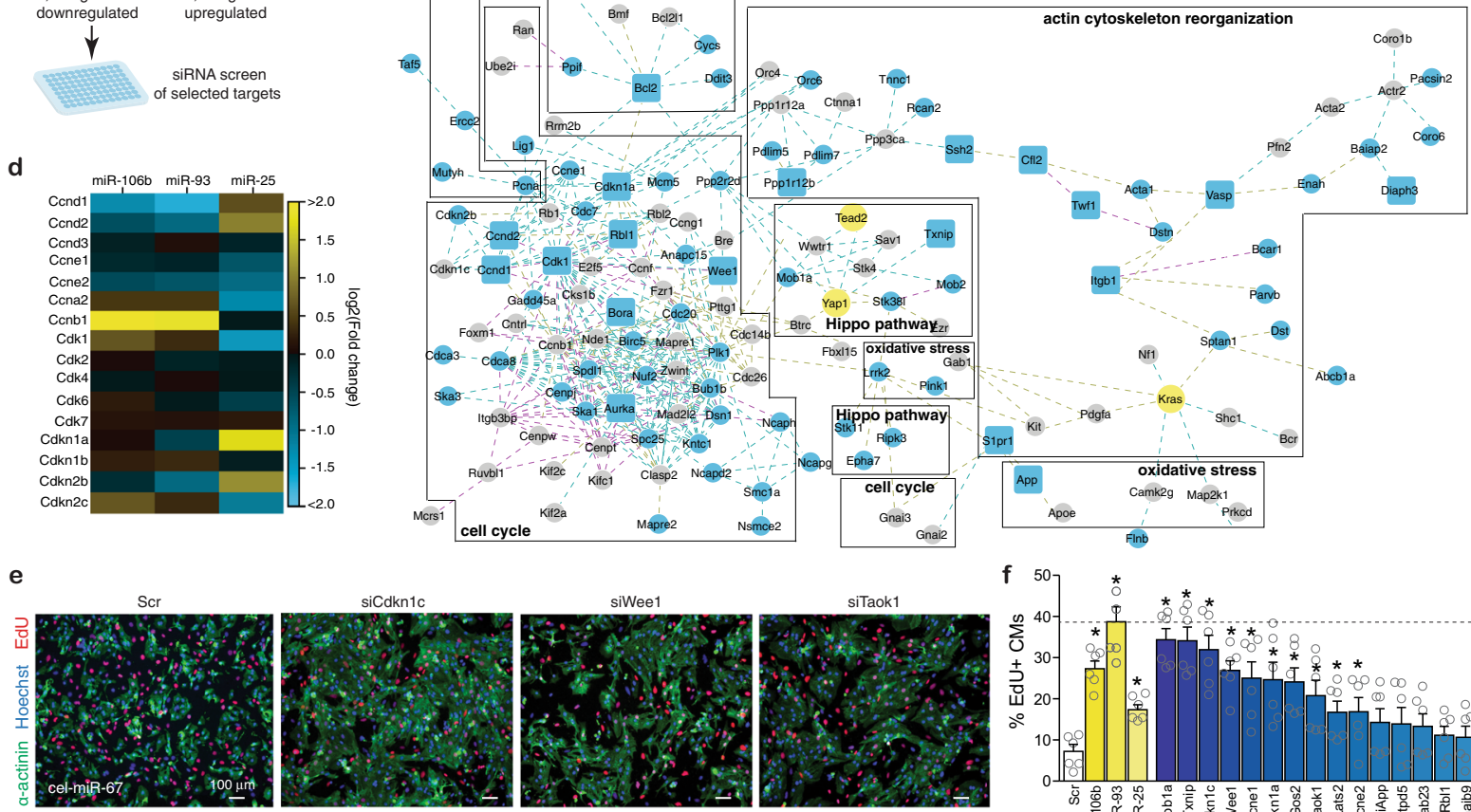

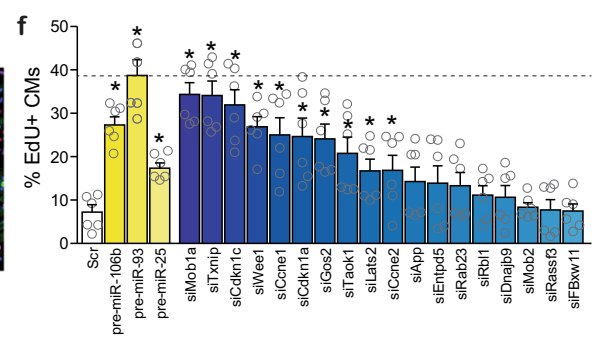

Fig. 5 The miR-106b 25 cluster suppresses cell cycle inhibitors. a Workflow for the experiment and miRNA target validation. $\mathbf{b}$ Venn diagram showing the relationship between the sets of downregulated genes with miR-106b, miR-93, or miR-25 seed regions and a 1.3-fold change cut-off with respect to cel-miR67. c A compartmentalized network of differentially expressed genes involved in cell cycle regulation. Each connection is color-coded according to the miRNA that regulates the differentially expressed gene (magenta for miR-106b, cyan for miR-93 and dark yellow for miR-25). Genes indicated by blue circles are downregulated by at least one miRNA while downregulated genes shown in blue squares are found to be bioinformatic targets by at least 1 miRNA. Genes represented by yellow circles are upregulated genes by all three miRNAs. $\mathbf{d}$ Heatmap representation of cell cycle regulators differentially expressed by miR-106b 25 relative to cel-miR-67. e EdU staining of rat CMs treated with selected siRNAs. $\mathbf{f}$ Percentage of proliferating rat CMs after siRNA treatment, mean \pm s.e.m. represents $n=6$ biologically independent wells examined over one independent experiment. ${ }^{\star} P<0.05$ vs corresponding control group (error bars are s.e.m.). Statistical analysis consisted of a two-tailed Student's t-test (f). Source data are provided as a Source data file.

deletion approach, $m i R-106 b \sim 25$ knockout mice show enhanced paroxysmal atrial fibrillation related to disruption of a paired-like homeodomain transcription factor 2 homeobox gene (Pitx2) driven mechanism that controls the expression of the $m i R-17 \sim 92$ and miR-106b 25 clusters ${ }^{51}$. In line, Pitx 2 lies in close proximity to a major atrial fibrillation susceptibility locus on human chromosome $4 \mathrm{q} 25$ identified in genome-wide association studies ${ }^{52}$. Taken together, exceptional scrutiny should be considered when designing silencing strategies to therapeutically intervene in $m i R$ 25 expression in heart disease.

The combined observations in this study suggest a model whereby defined orchestration of cell cycle regulators underlies the developmental cell cycle arrest of postnatal cardiomyocytes. Moreover, the characteristics of $m i R-106 b \sim 25$ expression in this developmental time frame and its targetome provides a mechanistic explanation for cell cycle exit toward the acquirement of the terminally differentiated phenotype. Hence, when $m i R-106 b \sim 25$ expression is higher, as is the case in the early postnatal heart, cell cycle inhibitors including E2f5, Cdkn1c, Cone1 and Wee1 are actively suppressed resulting in a proliferative state and cardiomyocyte hyperplasia, while differentiation programs elicited by Hand 2 and Mef2d are suppressed. Vice versa, in the overloaded or injured adult heart when $m i R-106 b \sim 25$ expression is lower, cell cycle re-entry is actively suppressed by the derepression of cell cycle inhibitors, and a prohypertrophic terminal differentiation program is promoted. Taking advantage of the regulatory function between cardiomyocyte hyperplasia and hypertrophy by viral gene delivery of $m i R-106 b \sim 25$ produced regeneration of the adult myocardium in response to chronic ischemic injury. Our data demonstrate that exploitation of conserved epigenetic molecular programs can enhance the regenerative capacity of the injured myocardium.

\section{Methods}

Human heart samples. Approval for studies on human tissue samples was obtained from the Medical Ethics Committee of the University Medical Center Utrecht, The Netherlands, and by the Ethical Committee of the University Hospital Hamburg, Germany (Az. 532/116/9.7.1991). All patients or their relatives gave written informed consent before operation. In this study, we included tissue from the left ventricular free wall of patients with end-stage heart failure secondary to ischemic heart disease. Control tissue was taken from the left ventricular free wall of refused donor hearts. Failing hearts were also obtained from patients undergoing heart transplantation because of terminal heart failure. Non-failing donor hearts that could not be transplanted for technical reasons were used for comparison. The donor patient histories did not reveal any signs of heart disease.

Mouse models. Mice homozygous null for the mirc3 cluster (miR-106b 25) located in intron 13 of the $M \mathrm{~cm} 7$ (minichromosome maintenance complex 


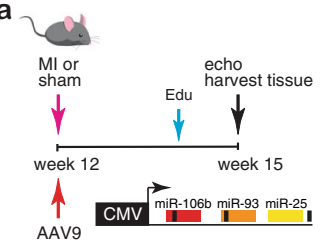

b

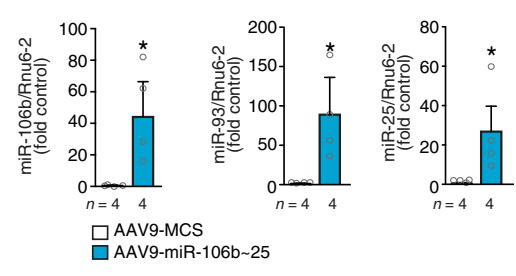

d

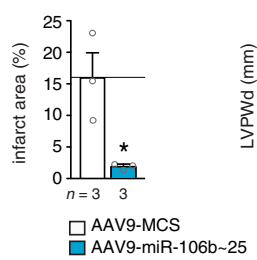

e

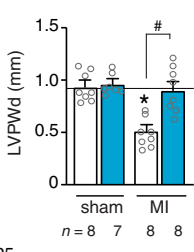

$\mathbf{f}$

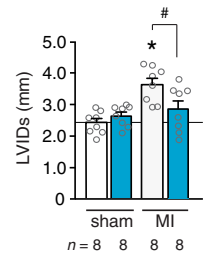

g

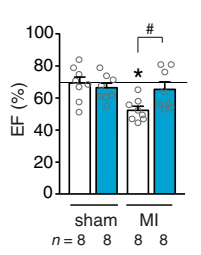

C

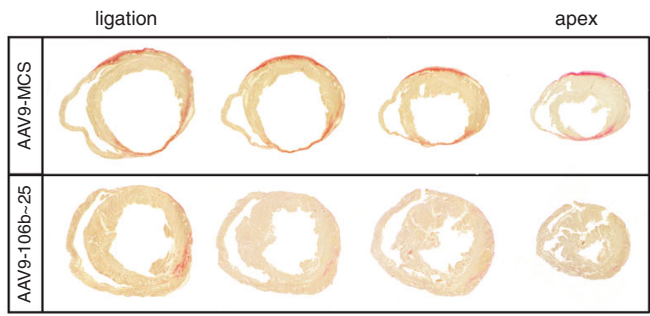

h
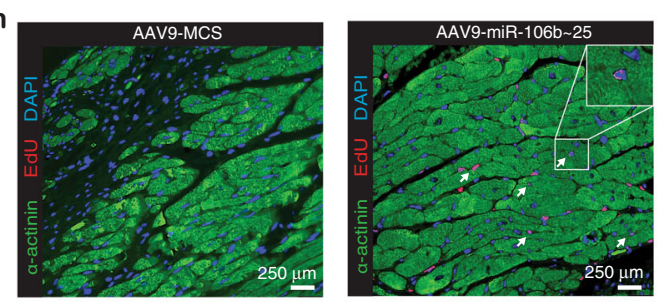
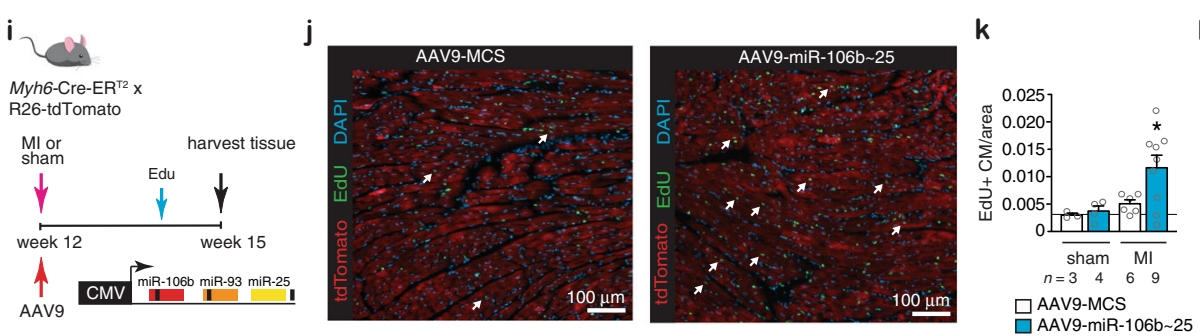

I

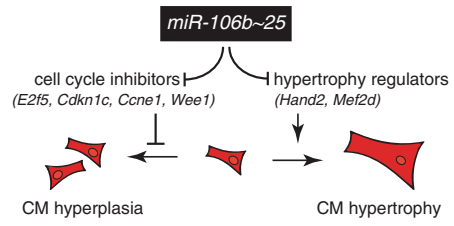

AAV9-miR-106b 25

Fig. 6 The miR-106b 25 cluster evokes myocardial regeneration. a Design of the study. b Real-time PCR analysis of miR-106b, miR-93 and miR-25 abundance in infarcted hearts receiving AAV9-MCS or AAV9-miR-106b 25. c Representative images of Sirius Red-stained ventricular cross-sections from the point of ligation toward the apex of hearts post-MI, treated with AAV9-MCS or AAV9-miR-106b 25. d Quantification of the infarct area of the postinfarcted left ventricles from mice receiving AAV9-MCS or AAV9-miR-106b 25. Quantification of e LVPWd, $\mathbf{f} L V I D s$, and $\mathbf{g}$ EF of mice that received AAV9-MCS or AAV9-miR-106b 25 after sham or MI surgery. i Representative images of the infarct border zone of mice treated with AAV9-MCS or AAV9-miR-106b 25 after MI surgery, and stained for $\alpha$-actinin and EdU; $n$ refers to the number of animals. $\mathbf{i}$ Design of the lineage tracing study. j TdTomato expression overlaps with 5-ethynyl-2'-deoxyuridine (EdU). k Quantification of TdTomato+, EdU+ CMs. I Schematic representation of the model. ${ }^{\star} P<0.05$ vs corresponding control group; ${ }^{\#} P<0.05$ vs corresponding treatment group (error bars are s.e.m.). Statistical analysis consisted of a twotailed Student's $t$-test $(\mathbf{b}, \mathbf{d})$ or a One-way ANOVA followed by Dunnett multiple comparison test $(\mathbf{e}-\mathbf{g}, \mathbf{k})$. Source data are provided as a Source data file.

Table 3 Morphometric and echocardiographic characteristics of mice subjected to sham or MI surgery and treated for 3 weeks with AAV9-MCS or AAV9-miR-106b 25.

\begin{tabular}{|c|c|c|c|c|}
\hline & Sham & & MI & \\
\hline$n$ & 8 & 8 & 8 & 8 \\
\hline LV mass (mg) & $84 \pm 3$ & $90 \pm 2$ & $88 \pm 5$ & $107 \pm 4^{\star \#}$ \\
\hline LV mass/BW $(\mathrm{mg} / \mathrm{g})$ & $3.2 \pm 0.2$ & $3.8 \pm 0.4$ & $5.2 \pm 0.4^{\star}$ & $6.4 \pm 1.0^{\star}$ \\
\hline IVSd (mm) & $0.79 \pm 0.03$ & $0.81 \pm 0.02$ & $0.75 \pm 0.03$ & $0.90 \pm 0.03^{\star \#}$ \\
\hline LVIDs (mm) & $2.42 \pm 0.13$ & $2.56 \pm 0.14$ & $3.55 \pm 0.20^{\star}$ & $2.79 \pm 0.26^{\#}$ \\
\hline LVPWd (mm) & $0.88 \pm 0.03$ & $0.86 \pm 0.05$ & $0.55 \pm 0.05^{\star}$ & $0.87 \pm 0.10^{\#}$ \\
\hline LVPWs (mm) & $1.16 \pm 0.05$ & $1.21 \pm 0.06$ & $0.69 \pm 0.07^{\star}$ & $1.05 \pm 0.13^{\#}$ \\
\hline $\mathrm{EF}(\%)$ & $69 \pm 4$ & $67 \pm 3$ & $52 \pm 2^{\star}$ & $65 \pm 4^{\star \#}$ \\
\hline FS (\%) & $33 \pm 3$ & $31 \pm 2$ & $22 \pm 1^{\star}$ & $31 \pm 3^{\star \#}$ \\
\hline $\begin{array}{l}\text { Data are expressed as means } \\
B W \text { body weight, } L V \text { left vent } \\
\angle V I D s \text { left ventricular internal } \\
\text { fractional shortening, } E / A D \\
\text { *Indicates } P<0.05 \text { vs sham } \\
\text { \#Indicates } P<0.05 \text { vs experir }\end{array}$ & $\begin{array}{l}\text { stole, } L V P \text { w left } \\
\text { rent with a }\end{array}$ & rior wall thickness at end-c & ventricular pos & $\begin{array}{l}\text { cular internal dimension at } \\
\text { ss at end-systole, EF ejectio }\end{array}$ \\
\hline
\end{tabular}


component 7) gene were generated previously ${ }^{21}$, obtained from the Jackson Laboratory (Mirc3tm1.1Tyj/J, Stock No: 008460) and maintained in a B6SV129F1 background. Both male and female miR-106b 25 null mice of 3-6 months of age were used in this study. Other mice used in this study were 3-6-month-old male calcineurin transgenic mice in a B6SV129F1 background expressing an activated mutant of calcineurin in the postnatal heart under control of the $5.5 \mathrm{~kb}$ murine myh6 promoter (MHC-CnA) ${ }^{53}$; male and female $\mathrm{CD} 1$ wild-type mice ranging between postnatal (p) day 0 and p56; male and female B6SV129F1 wild-type mice of 3-6 months of age (Charles River Laboratories). Cre-responsive Rosa26TdTomato (R26-TdT) reporter mice ${ }^{54}$ were crossbred with Myh6-mER-Cre-mER mice ${ }^{55}$ to generate Myh6-mER-Cre-mER R26-TdT mice in a B6SV129F1 background and both male and female mice of 3-6 months of age were used. Tamoxifen dissolved in corn oil was administered prior to myocardial infarction $(20 \mathrm{mg} / \mathrm{kg})$. All animal studies were performed in accordance with local institutional guidelines and regulations and were approved by the animal review committee of Medanex Inc., the International Centre for Genetic Engineering and Biotechnology (ICGEB) and the University of Minnesota. Sample size was determined by a power calculation based upon an echocardiographic effect size. Randomization of subjects to experimental groups was based on a single sequence of random assignments. Animal caretakers blinded investigators to group allocation during the experiment and/or when assessing the outcome.

Production of recombinant AAV vectors. The precursors of $m m u-m i R-106 b$, $m m u-m i R-93$, and $m m u-m i R-25$ plus upstream and downstream flanking region sequences (total approximately 200 base pairs) were amplified from mouse genomic DNA isolated from a wild-type CD1 mouse heart, using QIAamp DNA mini kit (Qiagen), according to the manufacturer's instructions. The primers used to amplify the precursor sequences were: forward primer: $5^{\prime}$-GTATCATAAGGA TCCCTTTCCACTGCTCTGGTGAG-3' and reverse primer: $3^{\prime}$-GTATCATAAG TCGACCTCACCTAGCTGTCTGTCC-5' ${ }^{\prime}$. The amplified sequences were cloned into the pZac2.1 vector (Gene Therapy Program, Penn Vector core, University of Pennsylvania, USA) using the restriction enzymes BamH I and Sal I. Recombinant AAV serotype 9 vectors were generated at the AAV Vector Unit of ICGEB, Trieste (Italy), as described previously ${ }^{36}$. CD1 mice at postnatal day 1 were intraperitoneal injected with an empty AAV9 vector (AAV9-MCS; multiple cloning site, negative control) or AAV9-miR-106b 25 at a dose of $1 \times 10^{11}$ viral genome particles per animal, using an insulin syringe with 30 -gauge needle. 12 days after injection, the hearts were collected for histological analysis.

\section{Aortic banding, myocardial infarction, AAV9 delivery, and transthoracic} echocardiography. Transverse aortic constriction (TAC) or sham surgery was performed in 2-6 month-old B6SV129F1 mice by subjecting the aorta to a defined 27 gauge constriction between the first and second truncus of the aortic arch as described previously ${ }^{56,57}$. Myocardial infarction (MI) was produced in 2-6 monthold CD1 mice by permanent left anterior descending (LAD) coronary artery ligation $^{36}$. Briefly, mice were anesthetized with an intraperitoneally injection of ketamine and xylazine, endotracheally intubated, and placed on a rodent ventilator. Body temperature was maintained at $37^{\circ} \mathrm{C}$ on a heating pad. The beating heart was accessed via a left thoracotomy. After removing the pericardium, a descending branch of the LAD coronary artery was visualized with a stereomicroscope (Leica) and occluded with a nylon suture. Ligation was confirmed by the whitening of a region of the left ventricle, immediately post-ligation. Immediately after MI surgery, adult mice received an intracardiac injection of AAV9 vectors (AAV9-MCS or AAV9-miR-106b 25) at a dose of $1 \times 10^{11}$ viral genome particles per animal. 5ethynyl-2'-deoxyuridine (EdU, Life Technologies) was administered intraperitoneally $(500 \mu \mathrm{g}$ per animal) every 2 days, for a period of 10 days. For Dopplerechocardiography, mice were shaved and lightly anaesthetized with isoflurane (mean 3\% in oxygen) and allowed to breathe spontaneously via a nasal cone. Noninvasive, echocardiographic parameters were measured using a RMV707B (15-45 MHz) scan-head interfaced with a Vevo-770 high frequency ultrasound system (VisualSonics). Long-axis ECG-triggered cine loops of the left ventricular (LV) contraction cycle were obtained in B-mode to assess end-diastolic/systolic volume. Short-axis recordings of the LV contraction cycle were taken in M-mode to assess wall thickness of the anterior/posterior wall at the mid-papillary level. Doppler was used to determine the ratio between early (E) and late (A) ventricular filling velocity (E/A ratio) and to calculate the pressure gradient between the proximal and distal sites of the transverse aortic constriction and only mice with a pressure gradient $>50 \mathrm{~mm} \mathrm{Hg}$ were included. From B-mode recordings, LV length from basis to apex, LV internal diameter in systole (LVIDs) and diastole (LVIDd) were determined. From M-mode recordings, LV posterior wall thickness in systole (LV PWs) and diastole (LV PWd) were determined. LV mass was calculated with the following formula: $\left(0.8^{*}\left(1.04^{*}(((\right.\right.$ LVIDd + LV PWd + IVSd $) \wedge 3)-(($ LVIDd $)$ $\wedge 3))+0.6)$; fractional shortening (FS) was calculated with the following formula: (LVIDd-LVIDs)/LVIDd*100). Ejection fraction (EF) was calculated as ((SV/Vd)

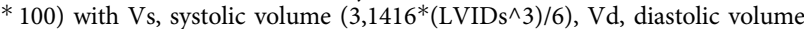
$\left(3,1416^{*}\left(\operatorname{LVIDd}^{\wedge} 3\right) / 6\right)$, and SV, stroke volume $(\mathrm{Vd}-\mathrm{Vs})^{57}$.

Fluorescent fluorescence activated cell sorting (FACS). Neonatal CD1 mice at age p1 randomly received AAV9-MCS or AAV9-miR106b 25. After 10 days, all mice we administered a single EdU injection and 2 days later cardiomyocytes were isolated and fixed using 4\% PFA. Next, cells were permeabilized using $0.1 \%$ Triton$\mathrm{X}$ and incubated for $2 \mathrm{~h}$ at room temperature with mouse monoclonal antibody [EA-53] to sarcomeric alpha-actinin (1:100, Abcam ab9465), followed by an incubation of $1 \mathrm{~h}$ with goat anti-mouse IgG secondary antibody Alexa Fluor-488 conjugated (1:100, ThermoFisher A-11001). Next, cells were further processed using the Click-IT EdU 647 Imaging kit to reveal EdU incorporation, according to the manufacturer's instructions, and stained with Hoechst 33342 (Life Technologies). Acquisition and analysis was performed on a BD FACSCelesta cell analyser. Analysis was performed using FACSDiva Version 6.1.3.

Histological analysis and (immunofluorescence) microscopy. Hearts were arrested in diastole, perfusion fixed with $4 \%$ paraformaldehyde/PBS solution, embedded in paraffin and sectioned at $4 \mu \mathrm{m}$. Paraffin sections were stained with hematoxylin and eosin (H\&E) for routine histological analysis; Sirius Red for the detection of fibrillar collagen; and FITC-labeled rabbit polyclonal antibody against wheat-germ-agglutinin (WGA) to visualize and quantify the myocyte crosssectional area (1:100, Sigma Aldrich T4144). Cell surface areas and fibrotic areas were determined using ImageJ imaging software (http://rsb.info.nih.gov/ij/). For immunofluorescence, paraffin sections were deparaffinized, rehydrated, and permeabilized with $0.5 \%$ Triton X-100/PBS, followed by overnight incubation at $4{ }^{\circ} \mathrm{C}$ in $1 \%$ BSA with primary antibodies: mouse monoclonal antibody [EA-53] to sarcomeric alpha-actinin (1:100, Abcam ab9465), rabbit polyclonal anti-phosphoHistone H3 (Ser10) (1:100, Sigma-Millipore 06-570), rabbit polyclonal anti-Aurora B (1:100, Abcam ab2254). Next, sections were washed with PBS and incubated for $2 \mathrm{~h}$ with goat anti-Mouse IgG Secondary Antibody Alexa Fluor-488 conjugated (1:100, ThermoFisher A-11001), donkey anti-rabbit IgG secondary antibody Alexa Fluor-555 conjugated (1:100, ThermoFisher A32794) or goat anti-mouse IgG secondary antibody Alexa Fluor-647 conjugated (1:100, Thermofisher A32728). For Edu staining sections were processed with the Click-IT EdU 555 Imaging kit to according the manufacturer's instructions. The nuclear counter-staining was performed with Hoechst 33342 (Life Technologies) and slides were then mounted in Vectashield (Vector Labs). Slides were visualized using a Zeiss Axioskop 2Plus with an AxioCamHRc.

Quantification of cardiomyocytes and binucleation. Procedures were described in detail previously ${ }^{24,26}$. Hearts were injected with $4 \mathrm{M} \mathrm{KCl}$ in the $\mathrm{LV}$ for cardioplegia, cut into 1-2 mm pieces and transferred to a peel-a-way embedding mold containing pre-warmed $8 \%$ gelatin in DPBS at $37^{\circ} \mathrm{C}$. Tissue was frozen using dryice chilled isopentane and stored at $-80^{\circ} \mathrm{C}$. Forty $\mu \mathrm{m}$ sections were prepared using a cryostat and placed on positively charged glass slides, allowed to dry for $30 \mathrm{~min}$ at room temperature (RT). Cryosections were incubated for $40 \mathrm{~min}$ in pre-heated DPBS at $37^{\circ} \mathrm{C}$ to remove the gelatin, fixed using $4 \%$ formaldehyde in DPBS for $15 \mathrm{~min}$ at RT, washed twice in 3\% bovine serum albumin (BSA) in DPBS for 5 min and then permeabilized with $1 \%$ Triton X-100 for $25 \mathrm{~min}$ at RT. Sections were incubated with EdU reaction buffer and washed 5 times in DPBS for $3 \mathrm{~min}$. Blocking was done by incubating the tissue with $4 \%$ fetal bovine serum (FBS) in $1 \%$ BSA in DPBS for $1 \mathrm{~h}$ at RT, prior to incubation with rabbit polyclonal anti-PCM-1 antibody $(0.4 \mathrm{mg} / \mu \mathrm{l}$ in blocking solution, Sigma-Aldrich HPA023370) overnight, at $4{ }^{\circ} \mathrm{C}$, followed by incubation with goat anti-rabbit IgG secondary antibody Alexa Fluor-488 conjugated $(4 \mathrm{mg} / \mu \mathrm{l}$ in blocking solution, ThermoFisher A32731) for $3 \mathrm{~h}$. Cell membranes were stained and nuclei counterstained by incubating the sections with wheat germ agglutinin (WGA) $(50 \mu \mathrm{g} / \mathrm{ml})$ and $4^{\prime}, 6^{\prime}$-diamino-2-fenilindol (DAPI) $(10 \mu \mathrm{g} / \mathrm{ml})$ for $1 \mathrm{~h}$ at RT, respectively, and mounted in fluoroshield mounting media without DAPI and slides sealed using nail polish. Images of LV fragments were produced in a Leica SP8 STED laser scanning confocal microscope. PCM-1 signal was used to define the beginning and ending of Z-Stack, which varied depending on the penetrance of the PCM-1 antibody. Images were composed of an average number of 40-50 stacks, corresponding to a thickness of 20-25 $\mu \mathrm{m}$. Five images, from different fragments, were collected per heart. After image acquisition, images were quantified with assistance of FIJI and IMARIS. $\mathrm{PCM}-1+$, EdU + cells were considered when there was an overlap between PCM-1 (green), EdU (red) and DAPI (blue). Cardiomyocyte density and binucleation were determined for each individual heart by averaging the result obtained in the different fragments. The average number of nuclei per cardiomyocyte was calculated using the formula: average $\mathrm{CM}$ nuclei number $=2 \times(\%$ binucleation $)+1 \times(\%$ mononucleation).

Western blot analysis. Whole tissue or cell lysates were produced in $150 \mathrm{mM}$ $\mathrm{NaCl}, 50 \mathrm{mM}$ Tris-HCl, $5 \mathrm{mM}$ EDTA, $50 \mathrm{mM} \mathrm{NaF}, 1 \%$ Igepal $^{\mathrm{m}}, 0,05 \%$ SDS, $40 \mathrm{mM} \beta$-glycerophosphate, $10 \mathrm{mM}$ Na-pyrophosphate, PhosSTOP- and Protease inhibitor cocktail (Roche Applied Science). Samples were boiled in 4x Leammli buffer, including $2 \% \beta$-mercaptoethanol, for $5 \mathrm{~min}$ at $95^{\circ} \mathrm{C}$. SDS-PAGE and western blotting were performed using the Mini-PROTEAN 3 system (Biorad). Blotted membranes were blocked in 5\% BSA/TBS-Tween. Primary antibody labeling was performed overnight at $4{ }^{\circ} \mathrm{C}$ at a concentration of $2 \mu \mathrm{g}$ IgG per $7 \mathrm{~mL}$ blocking buffer. Antibodies used included were: rabbit polyclonal antibody anti-p57 (H-91) (1:500, SantaCruz sc-8298), rabbit polyclonal antibody anti-E2f5 (1:500, Abcam ab22855), rabbit polyclonal antibody anti-E2F5 (E-19) (1:500, SantaCruz sc-999) 
rabbit monoclonal antibody anti-Cyclin E1 (D7T3U) (1:500, Cell Signaling Technology \#20808), rabbit polyclonal antibody anti-Wee1 (1:500, Cell Signaling Technology \#4936), rabbit polyclonal antibody anti-Mef2d (1:500, Abcam ab104515), mouse monoclonal anti-GAPDH (1:5000, Millipore, MAB374 clone 6C5), mouse monoclonal anti-alpha-Tubulin (1:5000, Sigma-Aldrich T6074) rabbit polyclonal anti-Histone H3 (1:5000, Cell Signaling Technology 9715S) and the secondary polyclonal swine anti-rabbit immunoglobulins/HRP (1:10,000, DAKO P0399) and polyclonal rabbit anti-mouse immunoglobulins/HRP (1:10,000, DAKO P0161). Secondary HRP conjugated antibodies were applied for $1 \mathrm{~h}$ at room temperature. Following antibody incubation, blots were washed for $3 \times 10 \mathrm{~min}$ in TBS-Tween. Images were generated using Supersignal West Dura Extended Duration ECL Substrate (Pierce) and the LAS-3000 documentation system (FujiFilm Life Science). Stripping was performed with Restore Western blot stripping buffer (Pierce). Output intensities were normalized for loading.

Quantitative PCR. Total RNA ( $1 \mu \mathrm{g})$ was extracted using miRNeasy Mini Kit (Qiagen) and applied to either miR-based or mRNA based reverse transcription. For miR-based reverse transcription, total RNA was reverse transcribed using miRCURY LNA Universal cDNA synthesis kit (Exiqon) followed by Real-time PCR using predesigned miRCURY LNA PCR primer sets (Exiqon) and miRCURY LNA SYBR Green master mix, according to the manufacturer's instructions ${ }^{57}$. Expression was normalized to expression levels of 5S rRNA. For mRNA-based reverse transcription, total RNA was reverse transcribed using hexameric random primers. The housekeeping gene ribosomal protein L7 (RPL7) was used for normalization. Fold changes were determined using the $2^{-\Delta \Delta C T}$ method. Real-time PCR primer sequences used in the study are listed in Supplementary Table 1.

\section{Primary cardiomyocyte cultures and transfections. Cardiomyocyte cultures} were isolated by enzymatic dissociation of 1 day-old neonatal rat hearts and processed for immunofluorescence as described previously ${ }^{58}$. Neonatal cardiomyocytes were seeded on Primaria 384-well plates (for microscopy) or in Primaria $10 \mathrm{~cm}$ dishes (for western blotting) and one day later, cells were transfected with mimics (Life Technologies) of hsa-miR-106b-5p, hsa-miR-93-5p, hsa-miR-25-3p or cel-miR-67 as control (25 mM) using (Lipofectamine RNAiMAX, Life Technologies). Twenty-four hours after transfection, culture medium was replaced by fresh medium; and $52 \mathrm{~h}$ after plating $5 \mu \mathrm{M}$ 5-ethynyl-2'-deoxyuridine (EdU, Life Technologies) was added for $20 \mathrm{~h}$. For siRNA transfection, selected siRNAs (Dharmacon) at a final concentration of $50 \mathrm{nM}$ were transfected in cardiomyocytes seeded at $7.5 \times 10^{3}$ cells per well in collagen-coated black clear-bottom 384 -well plates (PerkinElmer) $^{36}$. Cells were fixed at $72 \mathrm{~h}$ after plating and processed for immunofluorescence.

Immunofluorescence and image acquisition of cardiomyocytes. Cells were fixed with $4 \%$ paraformaldehyde for 15 min, permeabilized with $0.5 \%$ Triton X-100 in phosphate-buffered saline (PBS) solution for $10 \mathrm{~min}$, followed by $30 \mathrm{~min}$ blocking in $1 \%$ BSA (Roche). Cells were stained overnight at $4{ }^{\circ} \mathrm{C}$ with mouse monoclonal antibody [EA-53] to sarcomeric alpha-actinin (1:100, Abcam ab9465), followed by a goat anti-mouse IgG secondary antibody Alexa Fluor-488 conjugated (1:100, ThermoFisher A-11001). Next, cells were further processed using the Click-IT EdU 555 Imaging kit to reveal EdU incorporation, according to the manufacturer's instructions, and stained with Hoechst 33342 (Life Technologies). Image acquisition was performed using an ImageXpress Micro automated high-content screening fluorescence microscope at 10x magnification; a total of 16 images were acquired per wavelength, well and replicate, corresponding to $\sim 2500$ cells analyzed per condition. Image analysis was performed using the 'Multi-Wavelenght Cell Scoring' application module implemented in MetaXpress software (Molecular Devices) ${ }^{36}$. Proliferating cardiomyocytes were identified by a positive signal for the proliferation marker EdU and a positive signal for sarcomeric $\alpha$-actinin.

Luciferase-reporter assays. Constructs bearing $309 \mathrm{bp}$ of murine E2f5 3'UTR (pMIR-E2f5), 591 bp of murine Cdkn1a 3'UTR (pMIR- Cdkn1a), 625 bp of murine Cdkn1c $3^{\prime}$ UTR (pMIR- Cdknlc) and 656 bp of murine Mef2d 3'UTR (pMIRMef2d) were subcloned into the pmirGLO Dual-Luciferase miRNA Target Expression Vector (Promega). Correspondent seed-sequence mutated Dual-pMIRreport plasmids were obtained using the QuikChange XL Site-Directed Mutagenesis Kit (Agilent Technologies). Low-passage COS7 cells were grown in DMEM (Invitrogen) supplemented with $10 \%$ FCS and seeded $\left(2.5 \times 10^{4}\right)$ in 48 -well plates and transfected at $50-60 \%$ confluence with a total of $100 \mathrm{ng}$ Dual-pMIR-report plasmids using X-tremeGENE 9 DNA Transfection Reagent (Roche), followed by transfection with mimics (Life Technologies) of hsa-miR-106b-5p, hsa-miR-93-5p, hsa-miR-25-3p or cel-miR-67 as control (25 mM) using Oligofectamine (Invitrogen). Firefly and Renilla luciferase activities were measured using the Dual Luciferase Reporter Assay System (Promega), according to the manufacturer's instructions.

Transcriptomic analysis and clustering of fold change expression. Deepsequencing of total RNA isolated from neonatal rat cardiomyocyte cultures was performed $72 \mathrm{~h}$ after transfection of mimics (Life Technologies) of hsa-miR-106b5p, hsa-miR-93-5p, hsa-miR-25-3p, or cel-miR-67 as control (25 mM) by IGA
Technology Services (Italy) ${ }^{36}$. RNA purity, integrity and concentration were determined using an Agilent 2100 Bioanalyzer (Agilent Technologies). Only RNAs with a RIN value $>7$ and an rRNA $28 \mathrm{~S} / 18 \mathrm{~S}$ ratio $>2$ were considered for sample preparation. Two $\mu \mathrm{g}$ of total RNA per sample was sequenced on an Illumina HiSeq2000. Two lanes in 7-plex were run obtaining 2 millions of single-reads per sample, 50-bp long. Real-time image analysis, base calling, de-multiplexing, and production of FASTQ sequence files were performed on the HiSeq2000 instrument using the HiSeq Software. Raw sequence files were quality checked using FASTQC software (www.bioinformatics.babraham.ac.uk/projects/fastqc) and trimmed to remove Illumina adaptor using Cutadapt software. The raw sequencing reads were then mapped to Ensembl Rattus norvegicus reference genome (GCA_000001895.4 Rnor 6.0.89.6) $)^{59}$ using STAR software. Rounded gene counts were normalized to RPKM (reads per kilobase of exon model per million mapped reads) using the $\mathrm{rpkm}$ function in the Bioconductor package edge $\mathrm{R}^{60}$. Genes with RPKM values greater than 2.00 in both miRNA and cel-miR-67 transfected rat CMs were considered as expressed genes. Fold changes were taken with respect to the expression upon cel-miR-67 transfection. Genes whose expression fold change were greater than 1.3 were considered as differentially expressed. The complete RNA-seq data sets from this study were deposited at the Gene Expression Omnibus (GEO) with accession number GSE178867.

\section{Bioinformatics analyses: clustering of fold change expression; pathway} enrichment analysis; target predictions; network analyses. The Pearson correlation between the log2-fold changes for all pairs of miRNA were calculated. Clustering was performed hierarchically using the average linkage criterion with a Euclidean distance metric as implemented in SciPy v0.18.1 (http://www.scipy.org). A dendrogram was then generated using the SciPy clustering package to visualize the arrangement of the resulting cluster. Statistically enriched pathways from the set of differentially expressed genes were determined using a hypergeometric distribution-based statistical method as implemented in the Database for Annotation, Visualization and Integrated Discovery (DAVID) Bioinformatics Resources 6.8. The calculated $P$-values were then corrected according to Benjamini-Hochberg procedure to control the false discovery rates arising from multiple testing. KEGG pathways with $P<0.01$ and Benjamini-Hochberg FDR $<0.05$ are considered as statistically significant. Since bioinformatic predictions of seed sequence interactions with rat transcripts are not available, we compiled a list of rat miRNA-gene interactions from mouse predictions. Predicted mouse gene targets of the seed sequences (corresponding to miRNA families) of miRNAs that belong to the miR$106 b \sim 25$ cluster were collected from TargetScanMouse Release 7.1. The scores were calculated to be the most efficient interaction between a mouse gene and a human miRNA in a given miRNA family as determined by the seed sequence. The mouse genes were then translated to its corresponding rat genes through homology using the HomoloGene database. The list of miRNA-gene interactions was filtered to only include genes that were downregulated by the miRNA upon transfection to CMs according to the transcriptomic data (397 downregulated bioinformatic targets by $m i R-106 b, 429$ by $m i R-93$ and 358 by $m i R-25$ ). A bioinformatic proteinprotein interaction network was derived from STRING database v10.5, which integrates and scores protein interactions across different evidence channels (conserved neighborhood, co-occurrence, fusion, co-expression, experiments, databases, and text mining) and combines the scores from these channels. Only those interactions solely involving rat proteins were considered. To obtain a high confidence interaction network, a cut-off score of 700 (out of 1000) on the combined score was imposed. For each miRNA, a subnetwork was extracted containing only the differentially expressed genes upon imposing a 1.3-fold-change cut-off and the genes of interest in this study. These subnetworks were then merged into a single multi-network wherein the gene components can now be connected by three interactions, one for each miRNA. To further elucidate the multi-network, we cataloged the set of genes that are involved to biological processes of interest using the EMBL annotations of gene ontologies. These biological processes include cell division (GO:0051301 and GO:0000086) with 212 annotated genes, actin cytoskeleton (GO:0015629, GO:0030036, GO:0031532, and GO:0008154) with 345 annotated genes and oxidative stress response (GO:0006979) with 143 annotated genes. Furthermore, knowing the importance of the Hippo signaling pathway in cardiomyocyte proliferation, we also cataloged the set of genes involved in the canonical ${ }^{6}$ (19 genes) and non-canonical ${ }^{62}$ (21 genes) pathways in which Yap can be phosphorylated. These gene sets were then used to extract a compartmentalized network of genes that are differentially expressed after transfection of the miR$106 b \sim 25$ cluster whose interactions can lead to cytokinesis. Network analysis was done using the NetworkX v1.11 package in Python while graph visualization was done using Cytoscape v3.4.0.

Statistics and reproducibility. Results shown for images or blots were repeated independently at least once with similar results. The results are presented as mean \pm standard error of the mean (SEM). Statistical approaches for bioinformatics analyses are described above. All other statistical analyses were performed using Prism software (GraphPad Software Inc.), and consisted of One-way ANOVA followed by Dunnet's multiple comparison test when group differences were detected at the $5 \%$ significance level, or Student's $t$-test when comparing two experimental groups. Differences were considered significant when $P<0.05$. 
Reporting summary. Further information on research design is available in the Nature Research Reporting Summary linked to this article.

\section{Data availability}

The data that support the findings in this study are available within the article and its Supplementary information files. Raw and processed RNAseq data generated in this study have been deposited at the GEO database under accession code: GSE178867. Published resources evaluated included HomoloGene NCBI database (https://www.ncbi. nlm.nih.gov/homologene) and STRING (https://string-db.org/). Any remaining raw data will be available from the corresponding author upon reasonable request. Source data are provided with this paper.

Received: 10 August 2017; Accepted: 28 July 2021; Published online: 10 August 2021

\section{References}

1. Sedmera, D. \& Thompson, R. P. Myocyte proliferation in the developing heart. Dev. Dyn. 240, 1322-1334 (2011).

2. Soufan, A. T. et al. Three-dimensional measurement and visualization of morphogenesis applied to cardiac embryology. J. Microsc. 225, 269-274 (2007).

3. Becker, R. O., Chapin, S. \& Sherry, R. Regeneration of the ventricular myocardium in amphibians. Nature 248, 145-147 (1974).

4. Flink, I. L. Cell cycle reentry of ventricular and atrial cardiomyocytes and cells within the epicardium following amputation of the ventricular apex in the axolotl, Amblystoma mexicanum: confocal microscopic immunofluorescent image analysis of bromodeoxyuridine-labeled nuclei. Anat. Embryol. (Berl.) 205, 235-244 (2002)

5. Gonzalez-Rosa, J. M., Martin, V., Peralta, M., Torres, M. \& Mercader, N. Extensive scar formation and regression during heart regeneration after cryoinjury in zebrafish. Development 138, 1663-1674 (2011).

6. Oberpriller, J. O. \& Oberpriller, J. C. Response of the adult newt ventricle to injury. J. Exp. Zool. 187, 249-253 (1974).

7. Porrello, E. R. et al. Transient regenerative potential of the neonatal mouse heart. Science 331, 1078-1080 (2011)

8. Puente, B. N. et al. The oxygen-rich postnatal environment induces cardiomyocyte cell-cycle arrest through DNA damage response. Cell 157, 565-579 (2014).

9. Heallen, T. et al. Hippo pathway inhibits Wnt signaling to restrain cardiomyocyte proliferation and heart size. Science 332, 458-461 (2011).

10. D'Uva, G. et al. ERBB2 triggers mammalian heart regeneration by promoting cardiomyocyte dedifferentiation and proliferation. Nat. Cell Biol. 17, 627-638 (2015).

11. Mahmoud, A. I. et al. Meis1 regulates postnatal cardiomyocyte cell cycle arrest. Nature 497, 249-253 (2013).

12. Malek Mohammadi, M. et al. The transcription factor GATA4 promotes myocardial regeneration in neonatal mice. EMBO Mol. Med 9, 265-279 (2017).

13. Aguirre, A. et al. In vivo activation of a conserved microRNA program induces mammalian heart regeneration. Cell Stem Cell 15, 589-604 (2014).

14. Tian, Y. et al. A microRNA-Hippo pathway that promotes cardiomyocyte proliferation and cardiac regeneration in mice. Sci. Transl. Med 7, 279ra238 (2015).

15. Haubner, B. J., Schuetz, T. \& Penninger, J. M. A reproducible protocol for neonatal ischemic injury and cardiac regeneration in neonatal mice. Basic Res Cardiol. 111, 64 (2016)

16. Li, F., Wang, X., Capasso, J. M. \& Gerdes, A. M. Rapid transition of cardiac myocytes from hyperplasia to hypertrophy during postnatal development. $J$. Mol. Cell Cardiol. 28, 1737-1746 (1996).

17. Bergmann, O. et al. Evidence for cardiomyocyte renewal in humans. Science 324, 98-102 (2009).

18. Maillet, M., van Berlo, J. H. \& Molkentin, J. D. Molecular basis of physiological heart growth: fundamental concepts and new players. Nat. Rev. Mol. Cell Biol. 14, 38-48 (2013).

19. Velazquez, E. J. et al. Coronary-Artery Bypass Surgery in Patients with Ischemic Cardiomyopathy. N. Engl. J. Med. 374, 1511-1520 (2016)

20. Dirkx, E. et al. Nfat and miR-25 cooperate to reactivate the transcription factor Hand2 in heart failure. Nat. Cell Biol. 15, 1282-1293 (2013).

21. Ventura, A. et al. Targeted deletion reveals essential and overlapping functions of the miR-17 through 92 family of miRNA clusters. Cell 132, 875-886 (2008).

22. Inagaki, K. et al. Robust systemic transduction with AAV9 vectors in mice: efficient global cardiac gene transfer superior to that of AAV8. Mol. Ther. 14, $45-53$ (2006).
23. Buja, L. M. et al. Characterization of a potentially reversible increase in betaadrenergic receptors in isolated, neonatal rat cardiac myocytes with impaired energy metabolism. Circ. Res. 57, 640-645 (1985).

24. Alkass, K. et al. No evidence for cardiomyocyte number expansion in preadolescent mice. Cell 163, 1026-1036 (2015).

25. Sampaio-Pinto, V. et al. Neonatal apex resection triggers cardiomyocyte proliferation, neovascularization and functional recovery despite local fibrosis. Stem Cell Rep. 10, 860-874 (2018).

26. Sampaio-Pinto, V. et al. Stereological estimation of cardiomyocyte number and proliferation. Methods 190, 55-62 (2021).

27. Senyo, S. E. et al. Mammalian heart renewal by pre-existing cardiomyocytes. Nature 493, 433-436 (2013).

28. Brodsky, V., Sarkisov, D. S., Arefyeva, A. M., Panova, N. W. \& Gvasava, I. G. Polyploidy in cardiac myocytes of normal and hypertrophic human hearts; range of values. Virchows Arch. 424, 429-435 (1994).

29. Olson, E. N. \& Schneider, M. D. Sizing up the heart: development redux in disease. Genes Dev. 17, 1937-1956 (2003).

30. Kim, Y. et al. The MEF2D transcription factor mediates stress-dependent cardiac remodeling in mice. J. Clin. Invest. 118, 124-132 (2008).

31. Busk, P. K. et al. Involvement of cyclin D activity in left ventricle hypertrophy in vivo and in vitro. Cardiovasc. Res. 56, 64-75 (2002).

32. Pasumarthi, K. B., Nakajima, H., Nakajima, H. O., Soonpaa, M. H. \& Field, L. J. Targeted expression of cyclin D2 results in cardiomyocyte DNA synthesis and infarct regression in transgenic mice. Circ. Res. 96, 110-118 (2005).

33. Chaudhry, H. W. et al. Cyclin A2 mediates cardiomyocyte mitosis in the postmitotic myocardium. J. Biol. Chem. 279, 35858-35866 (2004).

34. Cheng, R. K. et al. Cyclin A2 induces cardiac regeneration after myocardial infarction and prevents heart failure. Circ. Res. 100, 1741-1748 (2007).

35. Shapiro, S. D. et al. Cyclin A2 induces cardiac regeneration after myocardial infarction through cytokinesis of adult cardiomyocytes. Sci. Transl. Med. 6, 224ra227 (2014).

36. Eulalio, A. et al. Functional screening identifies miRNAs inducing cardiac regeneration. Nature 492, 376-381 (2012).

37. Wang, B. et al. miR-25 Promotes Cardiomyocyte Proliferation by Targeting FBXW7. Mol. Ther. Nucleic Acids 19, 1299-1308 (2020).

38. Heallen, T. et al. Hippo signaling impedes adult heart regeneration. Development 140, 4683-4690 (2013).

39. von Gise, A. et al. YAP1, the nuclear target of Hippo signaling, stimulates heart growth through cardiomyocyte proliferation but not hypertrophy. Proc. Natl Acad. Sci. USA 109, 2394-2399 (2012).

40. Xin, M. et al. Hippo pathway effector Yap promotes cardiac regeneration. Proc. Natl Acad. Sci. USA 110, 13839-13844 (2013).

41. Xin, M. et al. Regulation of insulin-like growth factor signaling by Yap governs cardiomyocyte proliferation and embryonic heart size. Sci. Signal 4, ra70 (2011).

42. Leach, J. P. et al. Hippo pathway deficiency reverses systolic heart failure after infarction. Nature 550, 260-264 (2017).

43. Ikeda, S. et al. Hippo deficiency leads to cardiac dysfunction accompanied by cardiomyocyte dedifferentiation during pressure overload. Circ. Res 124, 292-305 (2019)

44. Wahlquist, C. et al. Inhibition of miR-25 improves cardiac contractility in the failing heart. Nature 508, 531-535 (2014).

45. Pan, L. et al. MiR-25 protects cardiomyocytes against oxidative damage by targeting the mitochondrial calcium uniporter. Int J. Mol. Sci. 16, 5420-5433 (2015).

46. Yao, Y., Sun, F. \& Lei, M. miR-25 inhibits sepsis-induced cardiomyocyte apoptosis by targetting PTEN. Biosci. Rep. 38, BSR20171511 (2018)

47. $\mathrm{Li}, \mathrm{H}$. et al. Alteration in microRNA-25 expression regulate cardiac function via renin secretion. Exp. Cell Res. 365, 119-128 (2018).

48. Chiang, D. Y. et al. Loss of microRNA-106b-25 cluster promotes atrial fibrillation by enhancing ryanodine receptor type- 2 expression and calcium release. Circ. Arrhythm. Electrophysiol. 7, 1214-1222 (2014).

49. Thum, T. et al. Comparison of different miR-21 inhibitor chemistries in a cardiac disease model. J. Clin. Invest. 121, 461-462 (2011). author reply 462463.

50. Gebert, L. F. et al. Miravirsen (SPC3649) can inhibit the biogenesis of miR122. Nucleic Acids Res. 42, 609-621 (2014).

51. Wang, J. et al. Pitx2-microRNA pathway that delimits sinoatrial node development and inhibits predisposition to atrial fibrillation. Proc. Natl Acad. Sci. USA 111, 9181-9186 (2014)

52. Gudbjartsson, D. F. et al. Variants conferring risk of atrial fibrillation on chromosome 4q25. Nature 448, 353-357 (2007).

53. Molkentin, J. D. et al. A calcineurin-dependent transcriptional pathway for cardiac hypertrophy. Cell 93, 215-228 (1998).

54. Chen, Z. \& van Berlo, J. H. Genetic lineage tracing of non-cardiomyocytes in mice. Methods Mol. Biol. 2158, 323-336 (2021). 
55. Sohal, D. S. et al. Temporally regulated and tissue-specific gene manipulations in the adult and embryonic heart using a tamoxifen-inducible Cre protein. Circ. Res. 89, 20-25 (2001).

56. Armand, A. S. et al. Cooperative synergy between NFAT and MyoD regulates myogenin expression and myogenesis. J. Biol. Chem. 283, 29004-29010 (2008).

57. da Costa Martins, P. A. et al. MicroRNA-199b targets the nuclear kinase Dyrkla in an auto-amplification loop promoting calcineurin/NFAT signalling. Nat. Cell Biol. 12, 1220-1227 (2010).

58. De Windt, L. J., Lim, H. W., Haq, S., Force, T. \& Molkentin, J. D. Calcineurin promotes protein kinase $\mathrm{C}$ and $\mathrm{c}$-Jun $\mathrm{NH} 2$-terminal kinase activation in the heart. Cross-talk between cardiac hypertrophic signaling pathways. J. Biol. Chem. 275, 13571-13579 (2000).

59. Yates, A. et al. Ensembl 2016. Nucleic Acids Res. 44, D710-D716 (2016).

60. McCarthy, D. J., Chen, Y. \& Smyth, G. K. Differential expression analysis of multifactor RNA-Seq experiments with respect to biological variation. Nucleic Acids Res. 40, 4288-4297 (2012).

61. Yu, F. X. \& Guan, K. L. The Hippo pathway: regulators and regulations. Genes Dev. 27, 355-371 (2013).

62. Mohseni, M. et al. A genetic screen identifies an LKB1-MARK signalling axis controlling the Hippo-YAP pathway. Nat. Cell Biol. 16, 108-117 (2014).

\section{Acknowledgements}

E.D. is supported by a VENI award 916-150-16 from the Netherlands Organization for Health Research and Development (ZonMW), an EMBO Long-term Fellowship (EMBO ALTF 848-2013) and a FP7 Marie Curie Intra-European Fellowship (Project number 627539). V.S.P. was funded by a fellowship from the FCT/ Ministério da Ciência, Tecnologia e Inovação SFRH/BD/111799/2015. P.D.C.M. is an Established Investigator of the Dutch Heart Foundation. L.D.W. acknowledges support from the Dutch CardioVascular Alliance (ARENA-PRIME). L.D.W. was further supported by grant 311549 from the European Research Council (ERC), a VICI award 918-156-47 from the Dutch Research Council and Marie Sklodowska-Curie grant agreement no. 813716 (TRAIN-HEART).

\section{Author contributions}

E.D., A.R., S.O., and L.O. performed real time PCR experiments. A.R., H.A., and S.O. performed western blots. A.R. performed luciferase assays. E.D., C.T., and R.C. performed transcriptome analysis. H.A. and S.Z. performed surgical procedures in mouse models. E.D. performed echocardiography. E.D., A.R., V.S.P., and D.S.W. performed histology in mouse models. E.D. and A.R. analysed data. E.D. and S.M. performed FACS analyses. L.B. performed microscopic imaging. M.H., R.W., L.Z., D.N. J.V.B., and M.G. provided reagents and models. E.D., M.G. S.S., P.D.C.M., and L.D.W. designed the study. E.D. and L.D.W. wrote the manuscript. E.D, P.D.C.M., and L.D.W. acquired funding for the study. E.D. and A.R. contributed equally as joint first authors.

\section{Competing interests}

E.D., M.G., and L.D.W. filed the data in the manuscript for patent protection. P.D.C.M. and L.D.W. are co-founders and stockholders of Mirabilis Therapeutics BV. The remaining authors declare no competing interests.

\section{Additional information}

Supplementary information The online version contains supplementary material available at https://doi.org/10.1038/s41467-021-25211-4.

Correspondence and requests for materials should be addressed to L.J.D.W.

Peer review information Nature Communications thanks the anonymous reviewers for their contribution to the peer review of this work. Peer reviewer reports are available.

Reprints and permission information is available at http://www.nature.com/reprints

Publisher's note Springer Nature remains neutral with regard to jurisdictional claims in published maps and institutional affiliations.

cc) (i) Open Access This article is licensed under a Creative Commons Attribution 4.0 International License, which permits use, sharing, adaptation, distribution and reproduction in any medium or format, as long as you give appropriate credit to the original author(s) and the source, provide a link to the Creative Commons license, and indicate if changes were made. The images or other third party material in this article are included in the article's Creative Commons license, unless indicated otherwise in a credit line to the material. If material is not included in the article's Creative Commons license and your intended use is not permitted by statutory regulation or exceeds the permitted use, you will need to obtain permission directly from the copyright holder. To view a copy of this license, visit http://creativecommons.org/ licenses/by/4.0/

(C) The Author(s) 2021, corrected publication 2022 